\title{
Summary of Structural Material Modeling Development for the NEAMS Program in Fiscal Year 2020
}

\section{October 2020}

Idaho National Laboratory

B. W. Spencer

J. H. Ke

S. Biswas

W. M. Hoffman

A. M. Recuero

D. Schwen

L. B. Munday

S. L. Dhulipala

S. A. Pitts

A. Casagranda

Temple University

L. Liu

J. He

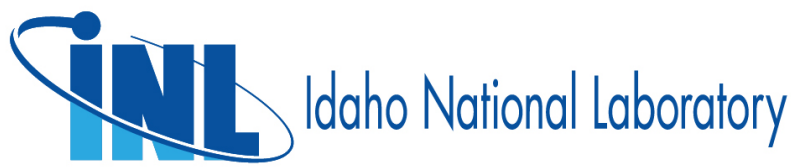




\section{DISCLAIMER}

This information was prepared as an account of work sponsored by an agency of the U.S. Government. Neither the U.S. Government nor any agency thereof, nor any of their employees, makes any warranty, expressed or implied, or assumes any legal liability or responsibility for the accuracy, completeness, or usefulness, of any information, apparatus, product, or process disclosed, or represents that its use would not infringe privately owned rights. References herein to any specific commercial product, process, or service by trade name, trade mark, manufacturer, or otherwise, does not necessarily constitute or imply its endorsement, recommendation, or favoring by the U.S. Government or any agency thereof. The views and opinions of authors expressed herein do not necessarily state or reflect those of the U.S. Government or any agency thereof. 


\title{
Summary of Structural Material Modeling Development for the NEAMS Program in Fiscal Year 2020
}

\author{
B. W. Spencer ${ }^{1}$ \\ J. H. $\mathrm{Ke}^{1}$ \\ S. Biswas ${ }^{1}$ \\ W. M. Hoffman ${ }^{2}$ \\ A. M. Recuero ${ }^{1}$ \\ D. Schwen ${ }^{1}$ \\ L. B. Munday ${ }^{1}$ \\ S. L. Dhulipala² \\ S. A. Pitts ${ }^{1}$ \\ A Casagranda ${ }^{1}$ \\ L. Liu $^{3}$ \\ J. $\mathrm{He}^{3}$
}

October 2020

${ }^{1}$ Computational Mechanics and Materials, Idaho National Laboratory

${ }^{2}$ Systems Integration, Idaho National Laboratory

${ }^{3}$ Mechanical Engineering, Temple University

Prepared for the

U.S. Department of Energy

Office of Nuclear Energy

Under DOE Idaho Operations Office

Contract DE-AC07-05ID14517 


\section{Contents}

1 Introduction $\quad 6$

2 Cluster Dynamics Modeling of Mn-Ni-Si Precipitation in RPV Steels 8

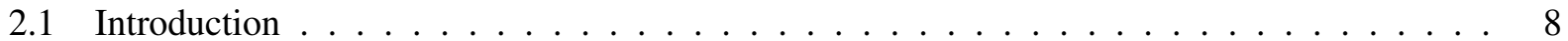

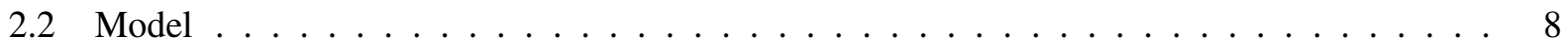

2.3 Grizzly Implementation . . . . . . . . . . . . . . . . . . . . . . . . 10

2.4 Sensitivity of MNSCD Fitting Parameters . . . . . . . . . . . . . . . 11

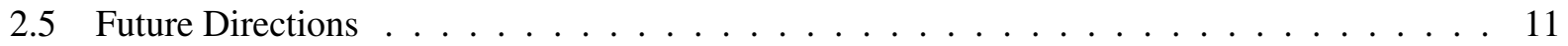

3 Improvements to Grizzly's Engineering-Scale Light-Water Reactor Pressure Vessel Analysis Capabilities 14

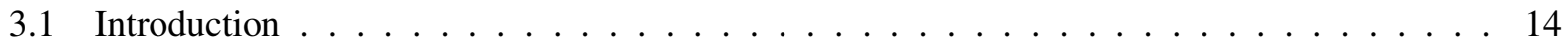

3.2 Performance Improvements for 2D and 3D Probabilistic Fracture Mechanics . . . . . . . 15

3.3 Improved Stress Intensity Factor Influence Coefficients for Embedded Flaws . . . . . . . . 19

3.4 Summary and Future Work . . . . . . . . . . . . . . . . . . . . 21

4 Bond-Slip Model For Reinforced Concrete $\quad 22$

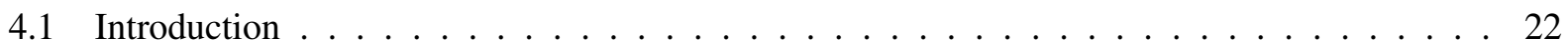

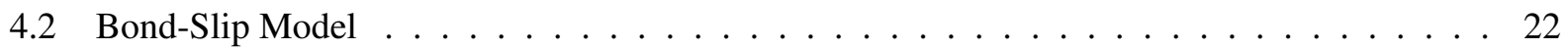

4.3 Model Verification . . . . . . . . . . . . . . . . . . . . 23

4.4 Beam Simulation Example . . . . . . . . . . . . . . . . . . . . . . . 24

4.5 Summary and Future Work . . . . . . . . . . . . . . . . . . . 25

5 Multiscale-Informed Modeling of High-Temperature Component Response with Uncertainty $\begin{array}{ll}\text { Quantification } & 26\end{array}$

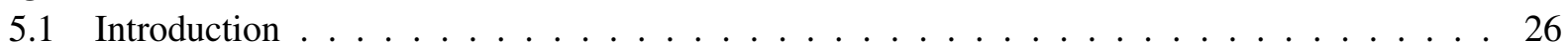

5.2 Creep Material Models . . . . . . . . . . . . . . . . . . . . . . 26

5.3 Simulation Results . . . . . . . . . . . . . . . . . . . . 27

5.4 Future Work . . . . . . . . . . . . . . . . . . . . . 28

6 Spectral Solver in the MOOSE Environment 29

6.1 Introduction . . . . . . . . . . . . . . . . . . . . . . . . . . . 29

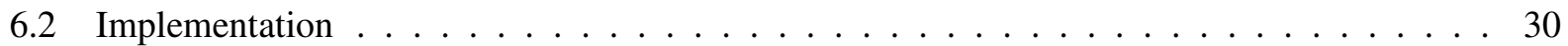

6.3 Numerical Results . . . . . . . . . . . . . . . . . . . . . . . . . 32

6.3.1 Numerical differentiation verification . . . . . . . . . . . . . . . 32

6.3.2 Solver verification - Diffusion problem . . . . . . . . . . . . . . . . 34

6.3.3 Solver verification - Linear elastic problem . . . . . . . . . . . . . . 36

6.3 .4 Computational efficiency . . . . . . . . . . . . . . . . 37 
6.4 Future Work . . . . . . . . . . . . . . . . . . . . 39

7 3D Non-Planar Crack Propagation with the Extended Finite Element Method 40

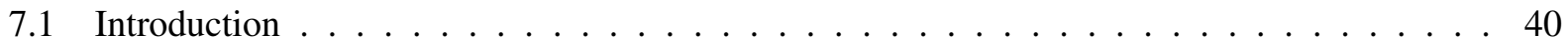

7.2 Non-Planar Crack Propagation $\ldots \ldots \ldots \ldots$. . . . . . . . . . . . . 40

7.3 Subcritical Crack Propagation and Life Prediction . . . . . . . . . . . . . . . . . 42

7.4 Benchmark Simulation: Growth of an Inclined Elliptical Crack . . . . . . . . . . . . . . . 43

7.5 Summary and Future Work . . . . . . . . . . . . . . . . . . 45

$\begin{array}{ll}\text { Acknowledgments } & 45\end{array}$

$\begin{array}{ll}\text { Bibliography } & 47\end{array}$ 


\section{Chapter 1}

\section{Introduction}

This report summarizes work performed during Fiscal Year (FY) 2020 at Idaho National Laboratory (INL) for the U.S. Department of Energy's Nuclear Engineering Advanced Modeling and Simulation (NEAMS) program for the Structural Materials and Chemistry Technical Area in the work package entitled "MS20IN050104 - Structural Materials - INL."

The Structural Materials and Chemistry Technical Area is a relatively new component of the NEAMS program and is currently focusing on developing simulation capabilities to support the deployment of nuclear energy in the areas of molten-salt reactor chemistry, light-water reactor (LWR) structural material degradation, and structural material behavior for advanced reactor applications. INL performed work to advance capabilities for the simulation of structural material behavior in both LWR and advanced reactor applications in the work described here.

Most of the capabilities documented here are being developed in the Grizzly code. Grizzly is a simulation code based on INL's MOOSE framework for modeling the processes and effects of structural material degradation for nuclear power plant components. It is based on the finite element method and is designed for multiphysics analysis.

The INL work in support of structural material modeling in FY 2020 was in the following areas, each of which had milestones or activities associated with them that were completed throughout the year.

- Evaluation of Precipitation Models for LWR Reactor Pressure Vessel (RPV) Steel Understanding the progression of embrittlement of RPV steel is critical for understanding the ability of RPVs to safely operate under long-term operation scenarios. One of the most important phenomena driving embrittlement is the formation and growth of manganese-rich precipitates. These are known as "lateblooming phases" because they only begin to appear later in the life of an RPV, which makes them particularly difficult to characterize. A previously-developed cluster dynamics model for the evolution of these precipitates was assessed here to determine what the most important sources of uncertainty in this model were and to develop a plan for future research in this area.

- Engineering-Scale LWR RPV Capability Development Grizzly has a maturing capability for the probabilistic fracture mechanics analysis of embrittled RPVs at the engineering scale. This is used to assess the likelihood that an RPV that has experienced embrittlement, due to exposure to service conditions, would fracture under the transient conditions that could occur during an accident. This work focused on further improving this capability, with a particular emphasis on improving the efficiency of these probabilistic calculations for three-dimensional simulations. This resulted in significant speedups for such simulations, making a class of simulations that previously incurred prohibitively high computational costs now feasible for routine use. A new form of reduced-order models used for fracture-mechanics computations was also implemented to reduce the conservatisms in the previous models. 
- Reinforced Concrete Mechanics Capability Development Another area where Grizzly has maturing capabilities is in the area of the multiphysics simulation of degradation in reinforced concrete. For this capability to be complete, it is important to have a comprehensive set of models for the nonlinear response of reinforced concrete under mechanical loading. Capturing the effects of bond slip between the rebar and concrete is an important part of such models. A bond-slip modeling capability that builds on a previously-developed system that enforced perfectly-bonded behavior between rebar and concrete has been developed here and tested on basic models as well as on a simulation of a mechanically-loaded reinforced concrete beam.

- Multiscale Modeling of High-Temperature Component Response with Uncertainty Quantification There are currently a large number of advanced reactor concepts under development, and most of these reactors operate at significantly higher temperatures than LWRs. This poses unique challenges on the metals exposed to this environment. Prior joint efforts between multiple national laboratories in this program have developed constitutive models to simulate creep in reactor metals under these conditions. This work integrated those models into Grizzly and performed an uncertainty quantification analysis to determine the effect of uncertain material parameters on the component-level response.

- Fast Fourier Transform (FFT) Solver for Periodic Microstructures in MOOSE A number of classes of simulations performed in codes based on MOOSE involve the simulation of periodic microstructures to determine the material-level response. These include spatially-resolved phase-field and polycrystal plasticity simulations. For such periodic microstructures, it is well known that FFTbased spectral solution approaches can provide significant performance improvements relative to the finite element approach that MOOSE is based on. An exploratory effort was performed here to develop an FFT solution strategy that can be used in the MOOSE platform. This will potentially allow for FFT-based solutions that leverage the significant set of physics models based on MOOSE.

- Propagation of Arbitrary Subcritical Cracks with the Extended Finite Element Method (XFEM) Fracture propagation is a significant issue for both LWR and advanced reactor structural component applications. A capability for arbitrary mesh-independent fracture growth based on XFEM has been under development for several years to enable such simulations. Previous efforts enabled the use of this for three-dimensional simulations but were limited to representing planar crack growth. This capability was generalized this year to permit the simulation of arbitrary non-planar cracks in three dimensions in MOOSE-based codes. For a number of important applications in nuclear power plant components, subcritical crack growth, such as that due to fatigue or stress-corrosion cracking, is also important, and options were added in the XFEM-based capability in MOOSE to permit that to be modeled. These foundational developments open up the possibility for modeling a large number of problems of interest for LWR and advanced reactor structure applications.

Details of these efforts are provided in the order in which they are listed in the following chapters. Summaries of these individual efforts, along with future directions for research in these areas, are also provided in the individual chapters. 


\section{Chapter 2}

\section{Cluster Dynamics Modeling of Mn-Ni-Si Precipitation in RPV Steels}

\subsection{Introduction}

Irradiation embrittlement of reactor pressure vessel (RPV) steels is one of the critical issues potentially limiting long term operation of LWR nuclear power plants. Exposure to neutron flux and elevated temperature during reactor operation makes the material progressively more brittle. Because of the extreme expense in replacement or remediation of an embrittled RPV, it is generally assumed that, if an unacceptable degree of embrittlement occurs, a reactor would no longer be operable. The radiation-enhanced formation of $\mathrm{Mn}-\mathrm{Ni}$-Si-rich precipitates (MNSPs), or the so-called late blooming phase, is an important causes of embrittlement [1]. The late-occurring nature of these phases makes it difficult to extrapolate future embrittlement based on experimental observations of the progression of embrittlement gathered from operating reactors. Models to characterize the evolution of MNSPs are therefore a critical component of a simulation capability to understand and predict embrittlement as a function of fluence, flux, and other conditions experienced by the material during irradiation.

The computational work performed here is based on cluster dynamics, a mean-field mesoscale model developed to simulate the nucleation and growth of MNSPs, including the contribution of T3 $\left(\mathrm{Mn}_{6} \mathrm{Ni}_{16} \mathrm{Si}_{7}\right)$ and T6 $\left(\mathrm{Mn}(\mathrm{Ni}, \mathrm{Si})_{2}\right)$ phases. The rate theory model is constructed by a large set of master rate equations. It tracks the evolution of cluster/precipitate size distribution or population as a function of dose or fluence. The rate equations consider multiple events of cluster reactions, including emission, dissociation, adsorption, and combination, thus providing the capability to study early-state and nm-scale nucleation processes. The approach is of particular interest for studying solute/defect clustering to large second-phase coarsening and has been utilized to model precipitation in RPV steels.

The work performed this year and discussed here includes: (1) completing the code-to-code comparison between the MNSCD model developed by Ke et al. [2] and a Grizzly implementation of that model and (2) reviewing the MNSCD model/code and identifying the sensitivity and individual effects of various physical parameters. To guide the directions for future research in this area, it is important to understand which of the parameters in that model have both a major effect on the predicted outcome and high uncertainty in their values.

\subsection{Model}

The cluster dynamics model implemented here overall follows the work done by Ke et al. [2], which is based on the general method developed by Slezov to characterize the kinetics of first-order phase transformations 
[3]. The model utilizes the concept of "effective monomer" to describe the cluster reaction events that involve the combined effect of $\mathrm{Mn}, \mathrm{Ni}$, and $\mathrm{Si}$ diffusion. In addition to homogeneous nucleation, the model also incorporates the heterogeneous contribution caused by the clusters generated in displacement cascades. For detailed model descriptions and parameters, please see Ref. [2]. Here we provide a brief overview of this model.

The cluster dynamics master equation describing the time evolution of MNSP cluster densities can be expressed as

$$
\frac{\partial f_{n}}{\partial t}=R_{\text {het }}+J_{n-1 \rightarrow n}-J_{n \rightarrow n+1}
$$

where $f_{n}$ is the concentration of the cluster of size $\mathrm{n}$ (with a total of $\mathrm{n}$ atoms), $R_{\text {het }}$ is the heterogeneous nucleation rate, and $J_{n-1 \rightarrow n}$ is the flux associated with the cluster reaction from the cluster of class $n$ - 1 to class $n$. The cluster flux can be described by

$$
J_{n \rightarrow n+1}=\omega_{n, n+1}^{+} f_{n}-\omega_{n+1, n}^{-} f_{n+1}
$$

Coefficients $\omega_{n, n+1}^{+}$are the effective rates of adsorption reactions for clusters of size $n$ to grow to size $n+1$, and coefficients $\omega_{n+1, n}^{-}$are the rates of emission reaction for clusters of size $n+1$ to shrink to size $n$. The same description applies similarly to the reaction flux $J_{n-1 \rightarrow n}$. For multicomponent precipitates and matrices, as in RPV steels, the effective adsorption rate can be given as

$$
\omega_{n, n+1}^{+}=\left[\sum_{i=1}^{j}\left(\frac{v_{i \alpha}^{2}}{\omega_{n_{i}, n_{i}+1}^{+}}\right)\right]^{-1}
$$

where $j$ is the total number of solute species in precipitates, $v_{i \alpha}$ is the composition of species $i$ in precipitate phase $\alpha$, and $\omega_{n_{i}-1, n_{i}}^{+}$is the rate for each solute species $i$. For diffusion-controlled cluster reactions, the adsorption rate is derived as [3]

$$
\begin{gathered}
\omega_{n, n+1}^{+}=4 \pi c_{\beta} a_{\alpha} D_{\text {eff }} n^{1 / 3} \\
D_{\text {eff }}=\sum_{i=1}^{j}\left(\frac{v_{i \alpha}^{2}}{x_{i \beta} D_{i}}\right)
\end{gathered}
$$

where $c_{\beta}$ is the average concentration of the species in the matrix phase, which is the inverse of atomic volume $\Omega, a_{\alpha}$ is the lattice constant of the precipitate phase, $x_{i \beta}$ is the molar fraction of the species $i$ in the matrix phase, and $D_{i}$ is the diffusion coefficient of species $i$. Note that the diffusion coefficient in the model is scaled up to account for the radiation enhanced diffusion. The scaling method is based on the concept of effective fluence as defined as $\phi t_{e}=\phi t\left[\phi_{r} / \phi\right]^{p}$, where $\phi t$ is the actual fluence, $\phi$ is the dose rate (dpa/s), $\phi_{r}$ is an arbitrary reference dpa rate, and $p$ is the scaling factor, which is 0.2 for neutron irradiation in this model.

Coefficients $\omega_{n+1, n}^{-}$are the emission rate and can be described by the given relation with $\omega_{n, n+1}^{+}$

$$
\omega_{n+1, n}^{-}=\omega_{n, n+1}^{+} \exp \left(-\frac{\Delta G_{n}-\Delta G_{n+1}}{k T}\right)
$$

where $\Delta G_{n}$ is the formation energy of the cluster $n . G_{n}$ can typically be expressed as

$$
\Delta G_{n}=n\left(g_{p}-\sum_{i} x_{i \alpha} \mu_{i}\right)+4 \pi \sigma\left(\frac{3 \Omega}{4 \pi}\right)^{2 / 3} n^{2 / 3}
$$

where $g_{p}$ is the free energy per atom in the cluster phase, $x_{i \alpha}$ is the composition of the species $i$ in the precipitate phase $\alpha, \Omega$ is the atomic volume, $\mu_{i}$ is the chemical potential of species $i$ in the matrix, and $\sigma$ is the interface energy or surface tension. The formation energy is determined by the TCAL3 database based on the method of CALPHAD (CALculation of PHAse Diagrams). 


\subsection{Grizzly Implementation}

The MNSCD code is a cluster dynamics tool developed in Prof. Dane Morgan's group at the University of Wisconsin-Madison [2, 4]. The model was later implemented in the Grizzly application under the U.S. Department of Energy's Light Water Reactor Sustainability (LWRS) program in 2019 [5]. The code design and implementation were completed, while small discrepancies remained when compared to the MNSCD code.

The first phase of the present work was to evaluate the Grizzly implementation of that model to resolve those discrepancies. These were resolved by making some modifications to the MNSCDUpdate class in the Grizzly implementation of this model, which contains derived and continuously updated quantities, such as the cluster reaction fluxes between different sizes of clusters. The calculation of monomer concentrations in the matrix at each time step is corrected by summing the change of MNSP cluster density from the cluster size of 2 atoms. The resulting code-to-code verification demonstrates excellent agreement between MNSCD (CDUW) and Grizzly codes, as shown in Figures 2.1 and 2.2. The time evolution of T3 and T6 phase number densities and remaining solute concentration $(\mathrm{Mn}, \mathrm{Ni}$, and $\mathrm{Si}$ ) in the matrix calculated by Grizzly all agree with those calculated by the MNSCD code.

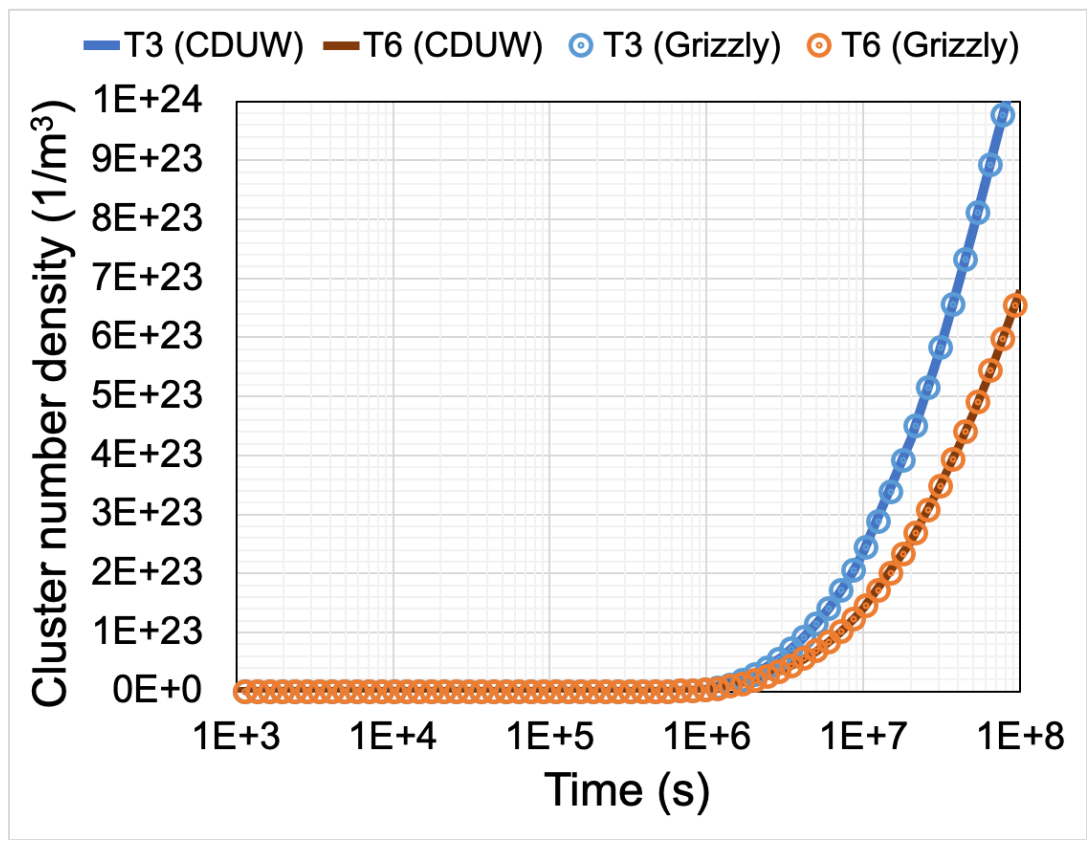

Figure 2.1: Time histories of T3 and T6 number densities from cluster dynamics simulations showing the comparison between those generated by the MNSCD code (CDUW) and Grizzly.

Despite the fact that the code-to-code benchmarking of the Grizzly implementation of the model shows that the model gives the correct behavior, the calculation for medium-large clusters $\left(>10^{4}\right)$ results in serious issues of speed and memory usage. The issues are caused by the large coupling Jacobian matrix that is implemented in Grizzly's MNSCDComposition class. A heap profiling performed using gperftools shows a significant amount of memory usage due to appending vectors, which appears to be an issue at the MOOSE framework level. Given that these issues make the Grizzly implementation struggle to handle the typical number of class or size of MNSPs, the Grizzly implementation of the MNSCD model is currently difficult to use for a sensitivity study before these issues are resolved. We plan to look into the issue in the future from the perspective of core components of MOOSE framework and to improve the speed and efficiency within the ODE solution subsystem. 


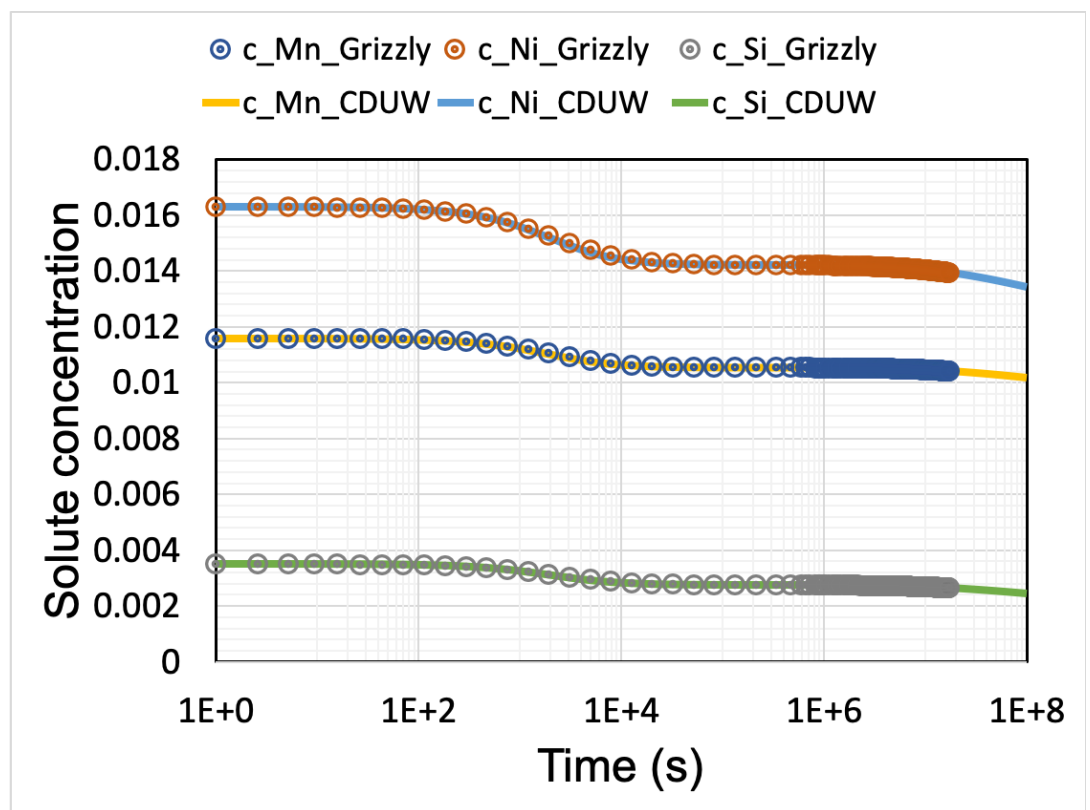

Figure 2.2: Time histories of $\mathrm{Mn}, \mathrm{Ni}$, and $\mathrm{Si}$ compositions in the matrix from cluster dynamics simulations showing the comparison between those generated by the MNSCD code (CDUW) and Grizzly.

\subsection{Sensitivity of MNSCD Fitting Parameters}

The MNSCD model has five major parameters that are fitted against available experimental data [2]. These parameters are the interface energies of $\mathrm{T} 3$ and $\mathrm{T} 6$ phases, the heterogeneous nucleation efficiency factor, the heterogeneous cluster size, and the scaling factor of radiation-enhanced diffusion or effective fluence. In the preliminary sensitivity analysis of their individual effects, it was found that, except for the heterogeneous cluster size, all of the other parameters have a strong effect on the evolution of the cluster size distribution. Additionally, these parameters are highly correlated. One example of this is the correlation between heterogeneous cluster size and interface energy.

Figure 2.3 shows the effect of heterogeneous cluster size (from 20 atoms to 200 atoms) on the size distribution of homogeneous and heterogeneous T3 clusters. The result shows that, as the heterogeneous cluster size becomes higher than 40 atoms, the overall result becomes insensitive to the adjusted parameter. This is because of the fact that 40 atoms may be close to the critical size of T3 cluster nucleation, which depends strongly on the interface energy and temperature. In other words, the best-fitted heterogeneous cluster size will increase as the temperature or T3 interface energy increases. These correlated fitting parameters and unsystematic data points may lead to the questionable extrapolation of the training data set, while the model indeed provides good fitting results for the interpolation of calibration data. It is therefore suggested to develop a model containing more specific physics, reduce the use of highly correlated fitting parameters, and utilize a data analytic approach to quantify the sensitivity and uncertainty of each fitting parameter.

\subsection{Future Directions}

One method to improve the MNSCD model is to replace some of the empirical fitting parameters with physics-based submodels. Defect evolution and radiation-induced segregation are critical physical events that have not yet been incorporated into this model. These two phenomena are highly correlated due to the complex chemical coupling between impurities, solute atoms, point defects, and other structural defects such 

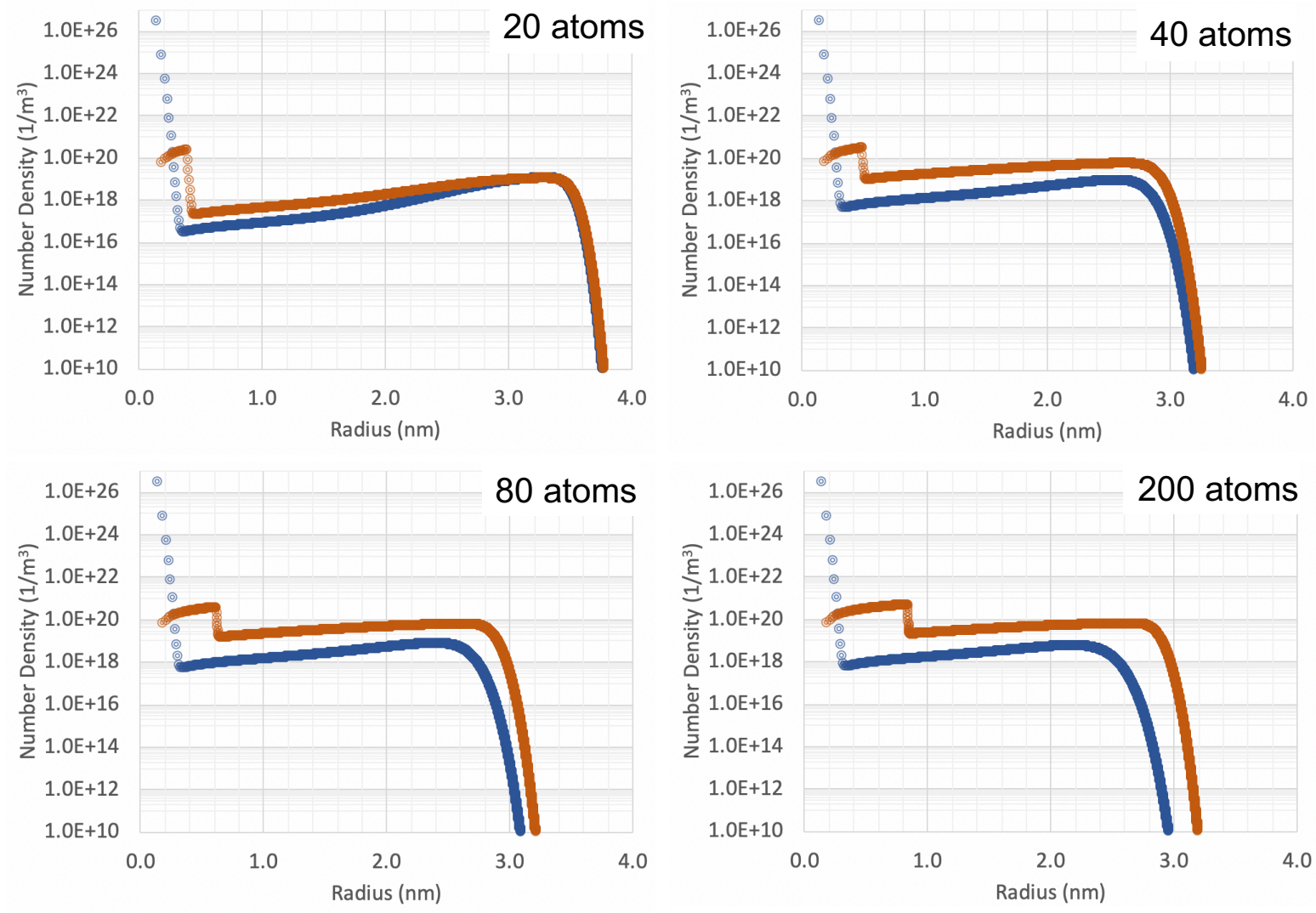

Figure 2.3: Comparison of homogeneous and heterogeneous T3 cluster size distributions calculated by using various heterogeneous cluster sizes, from 20 to 200 atoms. 
as sinks. They play important roles in modifying the thermodynamics of precipitate nucleation and solutedefect clustering, as well as diffusion-mediated processes accelerated by radiation. The models of defect evolution and solute segregation are often developed separately since they are best described by different types of master equations. Defect evolution, such as vacancy clustering, is usually modeled by rate theory equations characterizing multiple reaction events, while solute segregation modeling needs spatially-resolved reaction-diffusion equations with explicit local sink terms.

The cluster dynamics precipitation model [2] that is currently implemented considers a steady-state and semi-empirical model of radiation-enhanced diffusion (RED) developed by Odette et al. [6]. The RED model can be extended to involve radiation-induced unstable matrix features and impurity trapping into the rate theory based equations, but it is challenging to incorporate local solute/impurity segregation at dislocations or grain boundaries. The solute segregation was modeled separately and used to compute the local thermodynamic driving force of precipitation in cluster dynamics codes [7]. The model treated RED and solute segregation as independent events. Despite this approach providing reasonable agreement with experiments, the current rate-theory-based model can be improved significantly by adding a coupling of defect dynamics and segregation. The proposed direction conforms to the recent RPV steel review paper by Odette [8], commenting that $\mathrm{Cu}$-rich and $\mathrm{Mn}-\mathrm{Ni}$-Si precipitates can nucleate at heterogeneous sites, especially defect sinks such as dislocation loops and network dislocations. 


\section{Chapter 3}

\section{Improvements to Grizzly's Engineering-Scale Light-Water Reactor Pressure Vessel Analysis Capabilities}

\subsection{Introduction}

Since its inception, one of the major goals of Grizzly code development has been to develop improved capabilities for assessing the effects of structural component degradation on the ability of light-water reactor (LWR) nuclear power plants to safely operate under long-term operation (LTO) scenarios that could be well beyond their initial design lifetime. A major part of that effort has been on modeling the degradation of LWR reactor pressure vessels (RPV). This LWR RPV work has included the development of simulation capabilities both for the engineering-scale response of a degraded RPV under transient conditions as well as lower length scale simulation capabilities for predicting the microstructure evolution and the accompanying embrittlement that occur during long-term exposure to elevated temperature and radiation.

Both the engineering-scale and material-scale efforts are important to be able to assess the safety of an aged RPV. The engineering-scale work has focused on developing a modern, multidimensional, parallel scalable code that can address a wider variety of scenarios and do so more efficiently than existing tools. The material-scale work will provide embrittlement models that can be used for long-term aging with more confidence than existing models, which are based on experimental data of specimens exposed to shorter duration irradiation histories than those of interest for LTO. Grizzly currently uses semi-empirical embrittlement models for its engineering-scale analyses, but, when mature models based on the ongoing material-scale modeling efforts are available, the engineering-scale capabilities in Grizzly will provide a vehicle for them to be used for practical applications.

RPVs contain populations of flaws introduced during manufacturing. These flaws are typically small enough that they pose no safety concerns for the unirradiated RPV, but, after long term exposure to the operating environment, there is a potential concern that the age-related embrittlement could be sufficient to allow a crack to start propagating at the location of one of these flaws. To assess the effects of embrittlement on safety, probabilistic fracture mechanics (PFM) procedures are used in practice to compute the probability of fracture initiation or propagation through the RPV wall.

PFM analysis involves several steps:

1. Simulate the global thermomechanical response of the RPV under the transient loading scenarios of interest.

2. Generate a set of random realizations of a flaw population in the RPV according to known flaw densities 
for use in a Monte Carlo analysis.

3. Compute the stress intensity factor from the stress distribution in the global model, the local temperature, local embrittlement (using a spatial fluence distribution), and probability of fracture for every flaw in each realization.

4. Aggregate the results for all flaws to compute a conditional probability of fracture initiation (CPI) for the vessel given occurrence of the applied transient.

After several years of development, Grizzly has reached the point where it has a sufficiently complete feature set to perform a PFM analysis on degraded RPVs. These capabilities have been demonstrated, including benchmarking against the current state of the art code in this area (FAVOR [9]) for 1D representations of the RPV and a demonstration on a portion of an RPV in 3D for an idealized scenario [10].

The 2D and 3D capabilities in Grizzly are unique and have the potential to allow it to perform PFM calculations on a full RPV considering the effects of spatially varying geometry and coolant temperature, which cannot be addressed by existing codes. The current practice in most codes is to model the RPV using a 1D axisymmetric model, which assumes that the RPV behaves as an infinite cylinder. The aforementioned 3D demonstrations of Grizzly were limited to a small portion of the RPV because of significant performance issues in the initial 3D implementation. One of the main goals of the present work documented here is to address these performance issues to allow for efficient 3D modeling, which will allow for the novel modeling of full 3D RPVs, including coolant plume effects. As a result of this work, multidimensional (2D or 3D) PFM analysis is now orders of magnitude faster than it was previously and approaches the efficiency of 1D PFM analysis.

Because a large number of Monte Carlo iterations is required for convergence in these PFM calculations, a large number of evaluations of the mode- $I$ stress intensity factor, $K_{I}$, are required. Directly simulating these would be unfeasible, so reduced-order models are employed to allow for the rapid evaluation of $K_{I}$. There are separate models for the surface-breaking flaws and embedded flaws. The model for embedded flaws used by Grizzly has been used for a long time but is known to be conservative. The other major part of the present work was to implement an improved model that gives more realistic $K_{I}$ values in Grizzly.

Details on the development performed in these two areas are provided in the following sections.

\subsection{Performance Improvements for 2D and 3D Probabilistic Fracture Me- chanics}

As mentioned previously, the PFM calculation in Grizzly is run in a completely separate phase from the analysis of the global thermomechanical response. The thermomechanical analysis is a standard multiphysics finite element calculation in MOOSE, and, although 3D models can require significant computing resources as the model size increases, they offer good parallel scalability, and there are no major performance issues.

Although it is performed by Grizzly, the PFM calculation does not solve partial differential equations like a typical MOOSE solution does. Instead, it generates random samples to characterize the individual flaws and advances through time, evaluating local through-wall stress distributions, temperatures, local fluence, and $K_{I}$ for each flaw. The $K_{I}$ calculation is based on polynomial fits (up to 4th order) of the through-wall axial or hoop stress (depending on the flaw orientation) in the base metal and cladding on the interior of the RPV.

In 1D PFM simulations, polynomial coefficients describing the through-wall variation of stress and temperature are computed during the global thermomechanical analysis. MOOSE provides the ability to sample values of material properties or field variables at points sampled along a line using two different types of VectorPostprocessor objects: the LineMaterialSampler or LineValueSampler. The 


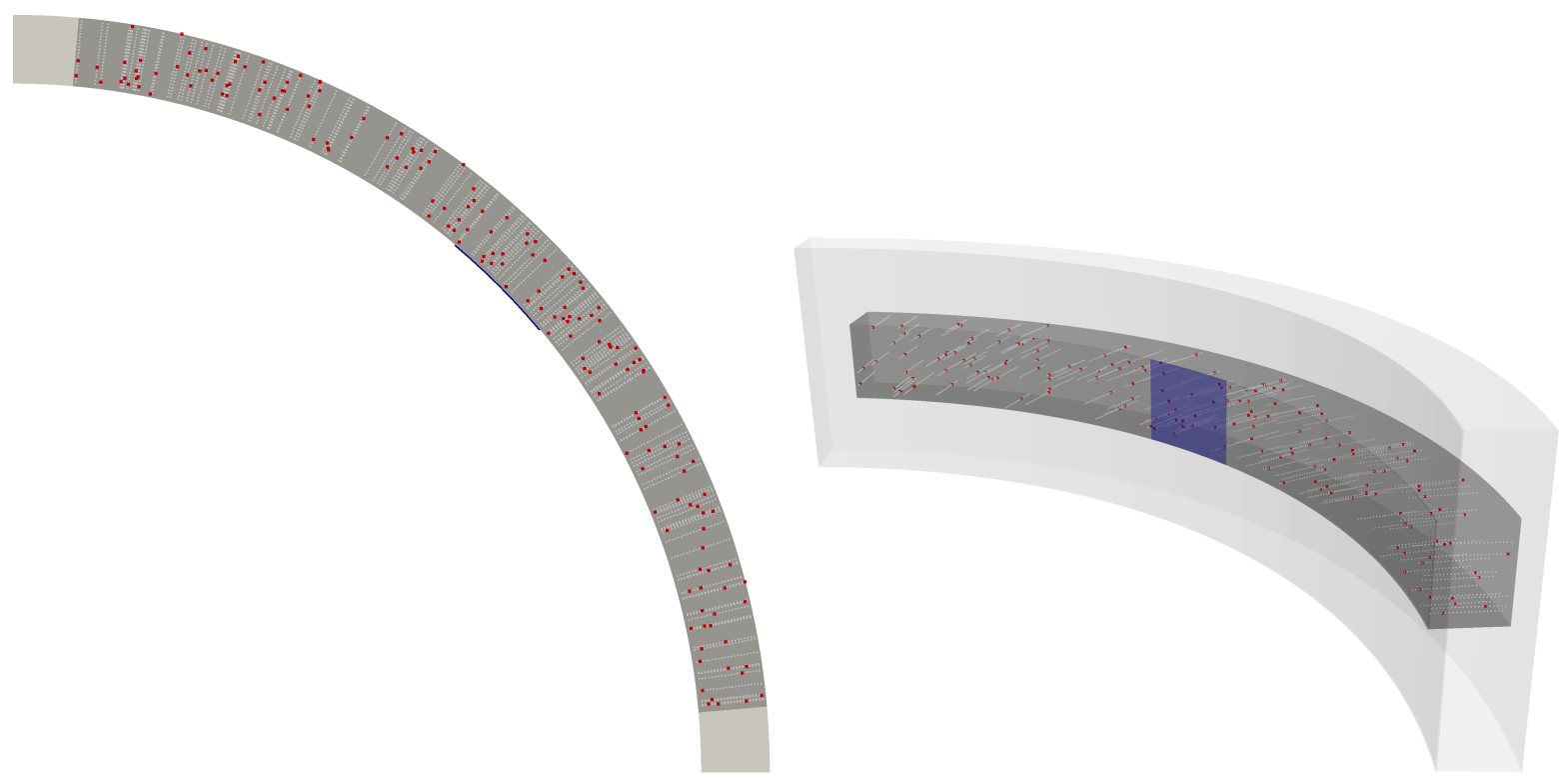

Figure 3.1: 2D (top) and 3D (bottom) models of a section of an RPV wall showing the locations of flaws (red dots) and points through the wall where solution fields are sampled from the ExodusII file to fit polynomials representing the through-wall variation of those fields, from [10].

LineValueSampler is used to extract the through-wall temperature distribution, while either one of these can be used for the stresses, with the appropriate options. Once the sampled values along a line are available, these values are fed into another VectorPostprocessor called LeastSquaresFitHistory, which performs a least-squares fit of a polynomial of arbitrary order for each of the quantities of interest and can output the history of the polynomials in tabular form in a comma-separated value (CSV) file.

For 3D calculations, on the other hand, previously the only way to output the spatially varying stresses and temperatures for use in the PFM calculation was through an ExodusII-formatted output file. ExodusII is the most commonly used format used for defining unstructured finite element meshes and mesh-based field data for the input and output of MOOSE codes.

Grizzly provides a flexible system for PFM calculations, as described in [10], which allows for different ways of performing the various parts of the PFM calculation to be implemented in a modular fashion. It provides options for obtaining the stresses and temperatures either from an ExodusII file (as field values) or from a CSV file (as polynomial coefficients). Previously, 1D calculations could read in the solutions using either the ExodusII file or CSV files, while 2D and 3D models were required to use ExodusII files.

When ExodusII files are used to transfer results between the global and PFM analyses, Grizzly samples the data at multiple points through the RPV thickness. This is shown in Figure 3.1, where the red dots represent the sampled flaw locations, and the lines of white dots represent the points where field values are sampled. The process of sampling field values from an unstructured mesh is inherently computationally expensive, especially for large 3D models, because, for each sampling point, the element containing that point must be located, which is nontrivial for meshes containing large numbers of elements.

Even though large numbers of flaws can be evaluated in parallel, and this process has been demonstrated to be highly scalable, finding a large number of values for points in an unstructured mesh for 3D models is computational prohibitive. Another issue with reading field values from the ExodusII file is that the stresses are highly discontinuous at the boundary between the base metal and cladding of the RPV, and outputting the field data in a way that correctly accounts for that discontinuity is problematic. There is an option to output 


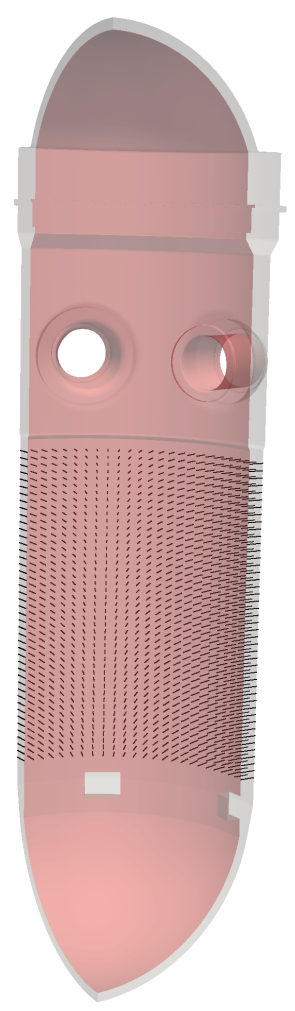

Figure 3.2: Global RPV model showing points used to sample solution fields (shown as black dots) through the depth on a uniform grid in the azimuthal and axial coordinates in the region of interest surrounding the core.

this data in a discontinuous manner, but it is much slower and results in a much larger output file than the continuous output.

To address these problems, a new scheme has been developed in which, in the PFM analysis, polynomial coefficients are read in from a CSV file, which is very similar to the way they are read in for a $1 \mathrm{D}$ global model. In the global 3D model, a regular grid of points is laid out on a roll-out of a large region of the RPV, and field values are sampled through the wall at those points to compute polynomial coefficients at those locations, as shown in Figure 3.2. Separate least-squares fits of the sampled variables at each of these points on the roll-out are performed, and the polynomial coefficients for each of these grid points is output to the CSV file.

Implementing this procedure in Grizzly involved adding a MOOSE Action that automatically creates samplers on this full grid, implementing a specialized version of the VectorPostprocessor that computes the least squares fit to perform multiple least-squares fits and expanding the previously-existing PolynomialCoefficientsFromFile object to allow it to read polynomial coefficients for this grid for 2D or 3D global models in addition to the 1D models that it previously supported.

Every flaw evaluated in the PFM analysis falls at some point within this grid, and the polynomial coefficients describing the through-wall variation of a field at that point are interpolated from those grid points to the current point. For any point in the vessel wall with a position defined by $r$, the radial (through-wall) position normalized by the wall thickness, $\theta$, the azimuthal coordinate, and $z$, the axial coordinate, a field 


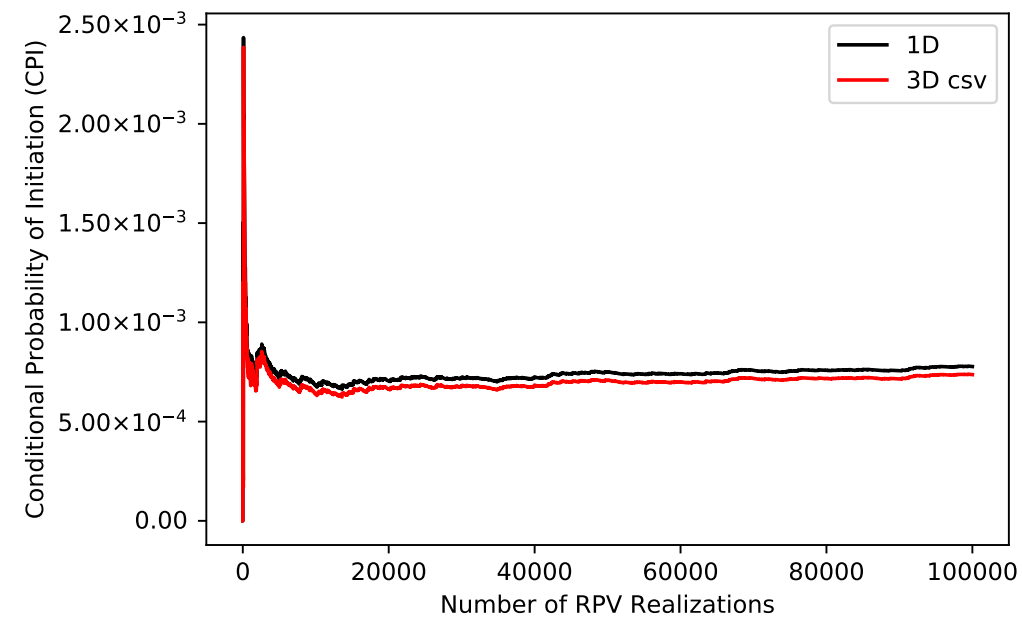

Figure 3.3: Convergence history of Monte Carlo PFM iterations for a demonstration PFM analysis.

quantity $\sigma$ can be expressed as:

$$
\sigma(r, \theta, z)=\sum_{i=1}^{4} \phi_{i}\left(C_{0 i}+C_{1 i} r+C_{2 i} r^{2}+C_{3 i} r^{3}+C_{4 i} r^{4}\right)
$$

where subscript $i$ denotes the index of the four points on the corners of the cell on the grid that contains the point, $\phi_{i}$ is an interpolation weight factor based on the proximity of the point to the $i$ th point on the cell that ranges from 0 to 1 , and $C_{0 i}$ through $C_{4 i}$ are the coefficients for the 4 th order polynomial fit at point $i$. Interpolated values of the individual polynomial coefficients are used in the $K_{I}$ calculation.

Although there is some computational expense involved in processing this expanded set of data in the CSV file (relative to the 1D case) and in performing the multidimensional interpolation of the polynomial coefficients, this is far less expensive than reading and sampling from ExodusII file results for 3D models. To quantify the speedup of this approach, the same PFM analysis of the prototypical RPV shown in Figure 3.2 was performed using a 1D and a 3D model of the RPV using the CSV grid approach and the ExodusII approach. Table 3.1 shows a comparison of the run times from the 1D CSV, 3D CSV, and 3D Exodus models, along with the computed values for CPI. It is typically necessary to run a large number (hundreds of thousands) of RPV realizations for the Monte Carlo iterations to converge, as shown in Figure 3.3, which shows the history of the mean CPI over the course of 100,000 Monte Carlo iterations for the case studied here. It is clearly necessary to run close to 100,000 iterations for convergence in this case, which was run using both 1,000 and 100,000 RPV realizations to facilitate performance comparisons. The 3D CSV case took about $3 \times$ longer than the 1D CSV case for both of these cases. The 3D Exodus case, took about $700 \times$ longer for the 1,000 realization case and ran out of compute time for the 100,000 realization case.

It should be noted that there is some additional computational burden associated with sampling the field values along the lines on the grid and performing least-squares fits in the global model. The run time for the global models used here increased about $43 \%$ relative to a baseline case when the additional line samplers were output. However, enabling discontinuous output, which is necessary for accurate results with the ExodusII file, increased the run time about $30 \%$ relative to the baseline case that only output continuous data. This slowdown is very small relative to the performance gains in the PFM stage of the analysis.

The computed CPI values are quite similar between the 1D and 3D CSV cases. Near the edges of the core, the stresses are actually somewhat lower than they are in the center of the core region, so a slightly lower CPI would be expected. The difference between the 3D Exodus and CSV results still requires some 
Table 3.1: Computation times and results for prototypical RPV PFM analysis.

\begin{tabular}{|l|l|l|l|l|}
\hline Num RPVs & Model & Run Time (s) & Processors & CPI \\
\hline \multirow{3}{*}{1,000} & 1D CSV & 26 & 180 & $7.79 \mathrm{e}-4$ \\
& 3D CSV & 81 & 180 & $7.39 \mathrm{e}-4$ \\
& 3D Exodus & 17,553 & 180 & $5.36 \mathrm{e}-4$ \\
\hline \multirow{3}{*}{100,000} & 1D CSV & 1,659 & 450 & $7.77 \mathrm{e}-4$ \\
& 3D CSV & 3,749 & 450 & $7.37 \mathrm{e}-4$ \\
& 3D Exodus & - & 450 & - \\
\hline
\end{tabular}

investigation.

Switching from reading fields from the Exodus file to interpolated polynomial coefficients from the CSV file clearly gives a significant speedup and enables computations that were not previously feasible. A firstof-its-kind simulation of a 3D RPV to demonstrate the effect of a region with lower coolant temperature near an inlet was recently performed using this capability for the LWRS program [11].

\subsection{Improved Stress Intensity Factor Influence Coefficients for Embedded Flaws}

To save computational effort in computing $K_{I}$, rather than directly performing calculations on detailed fracture models, Grizzly uses the influence coefficient procedure originally proposed by [12], which takes advantage of the fact that contributions to $K_{I}$ from the individual components of a polynomial expansion of the far-field stress applied to a crack can be linearly superimposed.

This technique represents the through-wall distribution of the stress component normal to the crack $\sigma$ as a function of $x$ and the through-wall position divided by the wall thickness, using up to a fourth order polynomial as:

$$
\sigma(x)=C_{0}+C_{1}(x)+C_{2}(x)^{2}+C_{3}(x)^{3}+C_{4}(x)^{4}
$$

where the $C_{i}$ terms are the polynomial coefficients of the stress distribution. $K_{I}$ can then be expressed as:

$$
K_{I}=\sum_{i=0}^{4} C_{i} K_{i} \sqrt{\pi a}
$$

where $a$ is the flaw dimension through the thickness of the wall. The $K_{i}$ coefficients are known as stress intensity factor influence coefficients (SIFICs) and are developed from fracture mechanics solutions.

Within their range of applicability, these SIFICs can provide highly accurate solutions. A variety of SIFICs have been used over the years for surface-breaking and embedded (subsurface) flaws. For surfacebreaking flaws, early versions of the FAVOR code used SIFICs interpolated from tables of values computed by the FAVOR developers using finite element models, but, more recently, high quality closed-form expressions for SIFICs for surface-breaking flaws have been made available in Article A-3000 of Section XI of the ASME Boiler \& Pressure Vessel Code. These have been adopted by Grizzly and FAVOR for such flaws.

For embedded flaws, Grizzly has used the procedure of FAVOR 16.1 documented in [9]. This does not use SIFICs but is based on the stresses at the tips of the flaw nearest to the inner and outer surface. Contributions from the bending and membrane components of the stress are superimposed, using correction factors that are shown graphically in Section XI, Article A-3000 of the 2013 and earlier versions of the ASME Boiler \& Pressure Vessel Code [13]. The procedure developed by [14] is used to analytically compute those correction factors. 

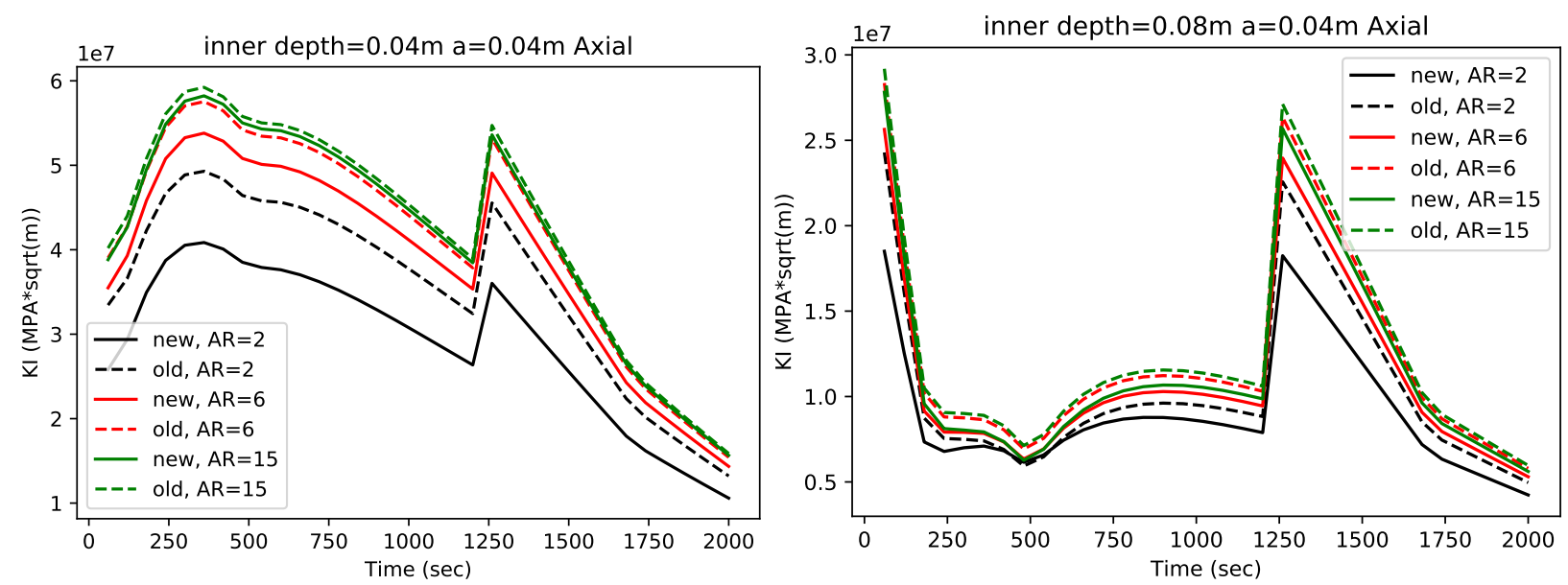

Figure 3.4: Time histories of $K_{I}$ computed using the new and old approaches for embedded axial elliptical flaws of various dimensions. $a$ is half the through-thickness dimension, and AR is the flaw aspect ratio.

Comparisons of the $K_{I}$ solutions obtained from this procedure with direct simulation results of the same flaw geometries in Grizzly have found that these solutions obtained from the SIFICs are significantly conservative. Starting with the 2015 version of the ASME Boiler \& Pressure Vessel Code, Appendix A-3000, Section XI has provided a new procedure for subsurface (embedded) flaws based on influence coefficients that was proposed in [15] and summarized in [16]. Limited direct finite element fracture solutions in Grizzly have shown very good comparison with these SIFICs.

To remove the excess conservatism in the older models, the new SIFIC-based approach for subsurface elliptical flaws, as described in the 2017 version of the ASME code [17], has been implemented as a new option in Grizzly. This uses tabular data that is interpolated in three dimensions as a function of the ratios $a / d, d / t$, and $a / l$, where $a$ is half the through-thickness flaw depth, $d$ is the depth to the center of the flaw from the nearest surface, and $l$ is the dimension of the flaw in the direction perpendicular to the thickness.

A set of SIFICs is provided in these tables for the inner, outer, and center points of the flaw. A small amount of manipulation of the tabular values is required to use them in Equation 3.3. SIFICs for up to 4th order polynomial representation of the flaws are provided in those tables for flaw depth to thickness $d / t$ ratios ranging from 0.2 to 0.5 . If the flaw is in the outer half of the RPV, the polynomial must be re-cast for a through-wall dimension starting from the outer wall. A closed-form procedure to do this has been implemented in Grizzly to allow it to use these SIFICs for flaws in both the inner and outer half of the RPV wall.

The fact that there are no SIFICs provided for $d / t<0.2$ is an important limitation of this approach because many of the critical flaws fall in the inner portion of the RPV wall that is outside this range, due to the fact that this area experiences high fluence and thermally driven stresses. Grizzly allows for a hybrid approach to be used, in which the new SIFICs are used in the regions where they are available and the old approach is used in the outer regions not covered by those SIFICs. More calculation of direct fracture solutions to assess the accuracy of these two approaches.

Figures 3.4 and 3.5 show time histories of $K_{I}$ computed for various flaw dimensions and depths in the axial and circumferential orientation using the new and old approaches described here. These are under the same idealized transient loading used to demonstrate the RPV PFM efficiency improvements. From these plots, it is evident that there is a decrease in $K_{I}$ for all cases and especially for those with high aspect ratios. 

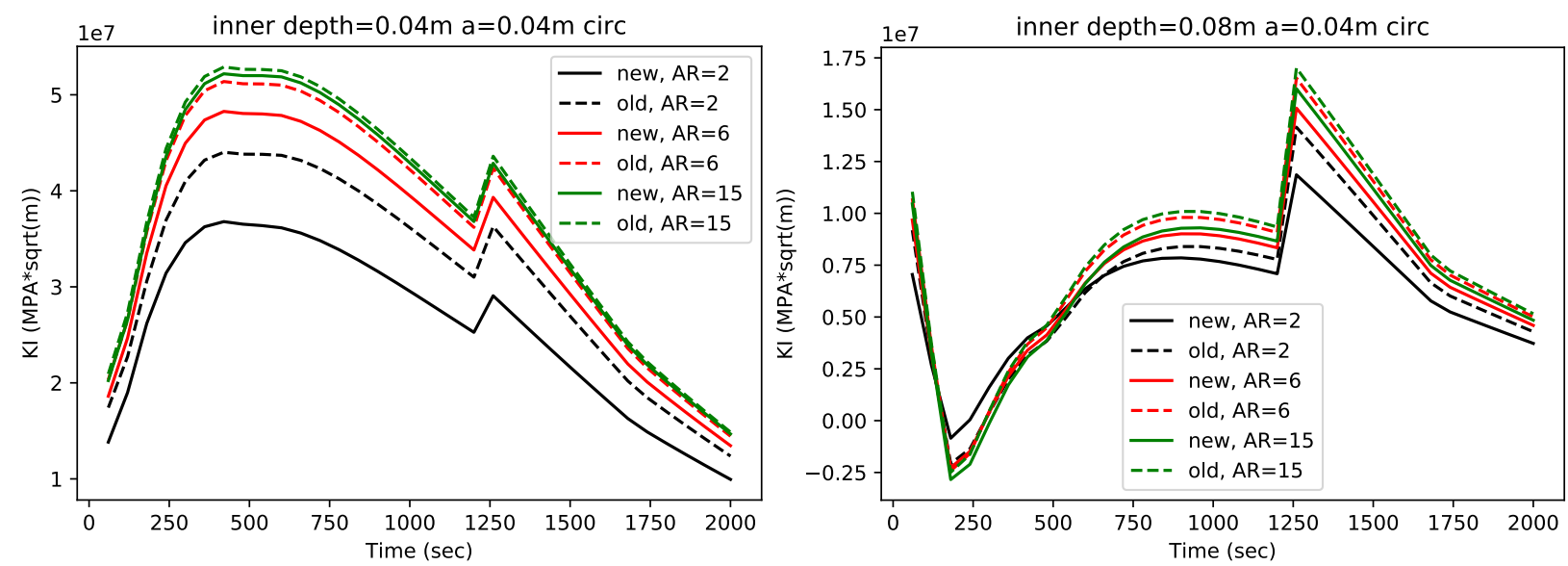

Figure 3.5: Time histories of $K_{I}$ computed using the new and old approaches for embedded circumferential elliptical flaws of various dimensions. $a$ is half the through-thickness dimension, and AR is the flaw aspect ratio.

\subsection{Summary and Future Work}

Two major improvements in Grizzly's PFM procedures for engineering analysis of embrittled LWR RPVs have been made here:

- A process that significantly improves the efficiency of PFM calculations based on 2D or 3D global RPV models has been developed and demonstrated. This procedure transfers polynomial coefficients for points on a grid between the global and PFM models and interpolates them, rather than extracting field data from an unstructured mesh. Speedups of greater than $700 \times$ were demonstrated using this new approach.

- A new SIFIC-based approach for computing $K_{I}$ for embedded flaws has been implemented in Grizzly and demonstrated to significantly remove excess conservatism in a selected set of cases.

These developments put Grizzly in a state where its higher-dimensionality features can be applied to real-world problems of interest. By coupling with a 3D computational fluid dynamics code, Grizzly can now be used to perform PFM analyses with much more realistic distributions of coolant temperature than have been used in the past.

The new embedded flaw $K_{I}$ calculation could potentially show that there are significant conservatisms in current PFM calculations. Once more testing is done to verify these models and compare them with the previous models, especially in the regions near the inner surface of the vessel, these will be used in PFM calculations to assess the effects of using models that have less conservatism built into them on the probabilistic quantities of interest. In addition, a recent paper [18] proposed a set of solutions for flaws near the vessel walls. After an evaluation of the accuracy of these models, these should potentially be included in the currently implemented models. 


\section{Chapter 4}

\section{Bond-Slip Model For Reinforced Concrete}

\subsection{Introduction}

Prior efforts over the past several years have incrementally developed a capability for modeling the progression of damage to reinforced concrete structures. A number of critical structures in both LWRs and advanced reactors are either reinforced or prestressed concrete and are subjected to the same types of degradation that can affect a variety of civil structures. In addition, select structures are exposed to high radiation, which presents unique issues.

The multiphysics solution capabilities in Grizzly/BlackBear are well-suited to the analysis of the progression of degradation because most of these mechanisms involve interactions between multiple transport mechanisms and mechanical deformation. One major area that is still missing important features is modeling the nonlinear mechanical response of concrete, rebar, and their interactions.

The primary focus this fiscal year has been on developing a bond-slip model for the connection between concrete and reinforcement bars, which is an important missing component of this nonlinear mechanical response modeling capability. Bond slip can have a major effect on the response of reinforced concrete under mechanical loading.

Rebar is modeled in Grizzly/BlackBear using one-dimensional line elements, while concrete is modeled using higher-dimensional solid elements. A constraint that rigidly ties the rebar nodes to the concrete elements that contain them was previously developed, which permits a flexible model definition because rebar elements need not coincide with the boundaries of concrete elements. An extension of this capability that permits slip between the rebar and concrete has been developed in BlackBear and demonstrated on both a basic pull-out test as well as on modeling of a large-scale concrete beam under bending.

\subsection{Bond-Slip Model}

The current work implements a simple engineering-scale bond-slip model that computes the bond stress based on the slip values calculated from the relative displacement between concrete and rebar. A node-toelement constraint is designed to apply the bond-slip relations. The implemented model follows the model proposed by Mehlhorn and Keuser [19]:

$$
\begin{aligned}
\sigma_{s} & =\sigma_{\max }\left[0.5\left(\frac{\Delta}{\Delta_{1}}\right)-4.5\left(\frac{\Delta}{\Delta_{1}}\right)^{2}+1.4\left(\frac{\Delta}{\Delta_{1}}\right)^{3}\right], \text { for } \Delta<\Delta_{1} \\
& =1.9 \sigma_{\text {max }}, \text { for } \Delta>=\Delta_{1}
\end{aligned}
$$

Here, $\sigma_{s}$ is the bond stress, $\sigma_{\max }$ is the maximum bond stress related to the compressive strength of the concrete, $\Delta$ is the slip calculated from the relative displacement between the concrete and rebar in the axial 
direction of the rebar, and $\Delta_{1}$ is the slip magnitude at which the maximum bond stress is reached. It should be noted that there are a number of other similar models for the relationship between bond stress and bond slip that could also be implemented in a similar way.

\subsection{Model Verification}

Figure 4.1 shows a simple pullout test used for the verification of the bond-slip model. The deformation is amplified by a factor of 1,000 for visualization purposes here.
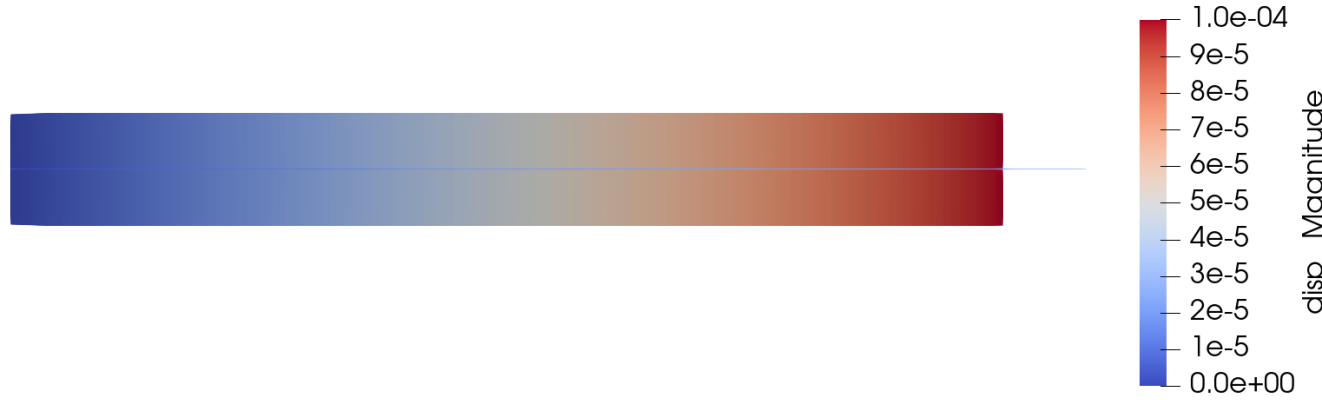

Figure 4.1: Rebar pullout test deigned for the verification of the bond-slip model. The deformation is amplified by a factor of 1,000 for visualization purposes.

Figure 4.2 demonstrates the bond-slip behavior obtained from the above mentioned pullout test. The model prediction here closely matches the experimental bond-slip behavior observed in [20]. It is also observed that the average stress-strain behavior of the rebar follows the same trend as the bond-slip model.

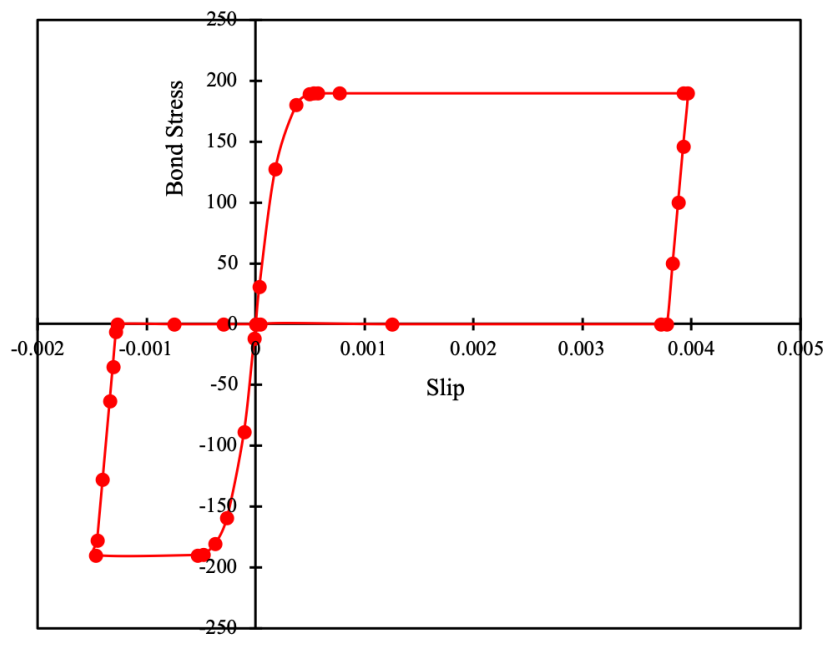

Figure 4.2: Bond-slip model implemented in BlackBear.

Next, we look at the influence of the new model on the axial stress compared to the equal value constraint that ties the rebar nodes to the adjacent concrete elements using a penalty factor. As observed in Figure 4.3, 
the bond-slip model introduces a nonlinear behavior in the rebar.

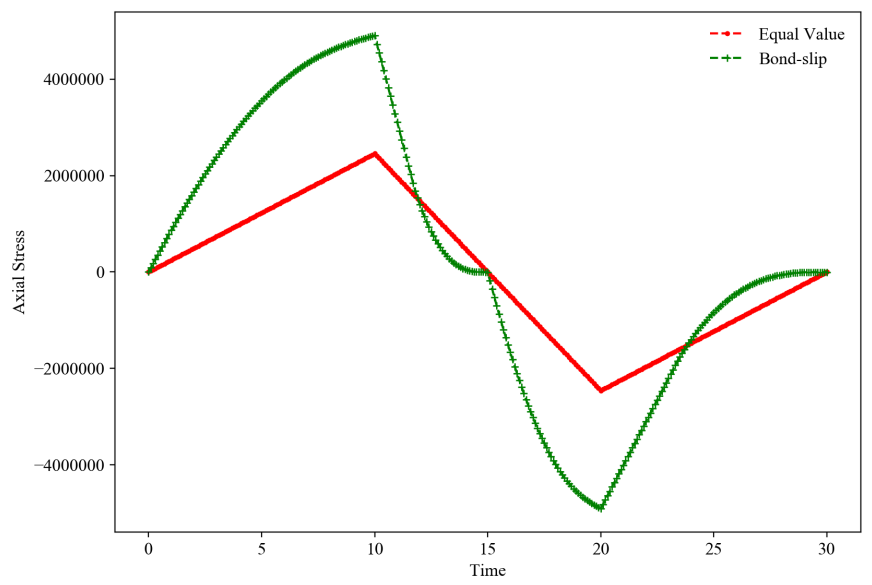

Figure 4.3: Average stress comparison between equal value constraint and bond-slip model.

Variation of average stress-strain behavior with maximum bond stress shows that a single order increase in the maximum bond stress increases the average stress of the rebar by several orders. This is demonstrated in Figure 4.4.

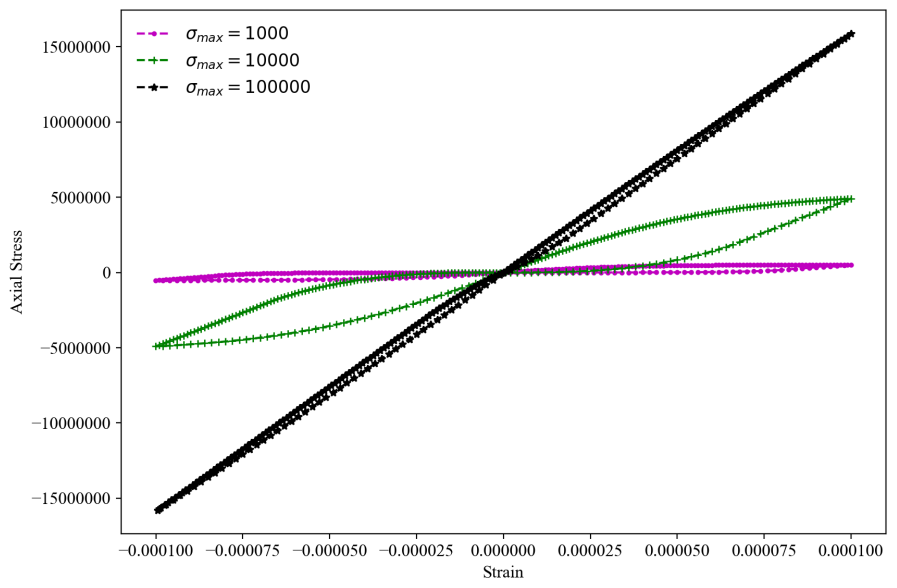

Figure 4.4: Average stress-strain behavior of the rebar during the pull-out test.

\subsection{Beam Simulation Example}

To demonstrate the bond-slip model on a larger-scale simulation, it was applied to a simulation of a large beam ( $6 \mathrm{~m}$ long $\times 1 \mathrm{~m}$ deep) that was loaded in bending up to the point of failure. The concrete in this beam is represented using $2 \mathrm{D}$ continuum elements. This beam only has a single layer of reinforcement along the bottom, represented using 1D line elements. The constraints between the rebar and concrete were represented in two different ways: using a tied constraint and using the bond-slip model described here. The problem is solved with plane strain assumptions, taking the incremental small-strain approach. The Mazars damage model was used for the concrete, while a simple linear elastic model was used for the rebar. 
Figure 4.5 shows the effect of the constraint on the damage pattern when the beam is loaded in bending. It compares the crack pattern formed in case of the bond-slip model with the crack formed with an equal value constraint. As observed in the figure, in both cases the crack initiates at the mid section of the beam and there is no significant difference between the failure modes. However, with the bond-slip model, the failure occurs faster. In case of the bond-slip model, the crack initiates at a midpoint displacement of 12.41 $\mathrm{mm}$, whereas with the equal value constraint, the crack initiates at a displacement $12.52 \mathrm{~mm}$. Moreover, the crack propagation happens faster in the former case that leads to the faster failure of the structure.
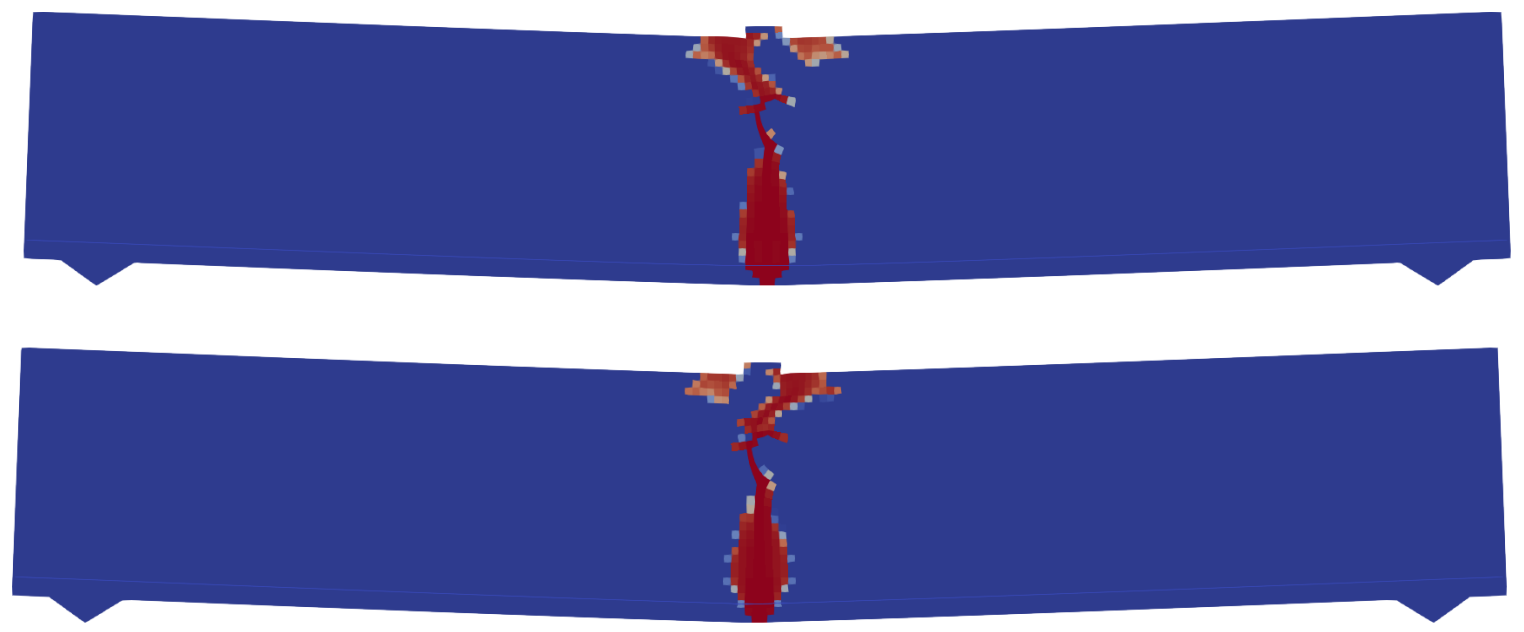

Figure 4.5: Failure of a reinforced beam in bending with a bond-slip constraint (top) and an equal value constraint (bottom).

This is an initial proof-of-concept simulation that is intended merely to demonstrate that the bond-slip model can be used on structural-scale simulations of interest. Further work is needed to validate simulation results using this model with experimental results.

\subsection{Summary and Future Work}

A basic bond-slip model has been implemented in BlackBear. Initial testing shows that this model behaves as expected, and it has been shown to be robust enough to be applied to a simulation at the scale of a structural member. Although we were able to perform initial demonstration simulations, this model still needs some additional work to improve its robustness on large-scale models. Further validation work of the model against laboratory tests are needed to demonstrate that it is accurately capturing these mechanisms.

An initial set of test cases that simulate the behavior of reinforced concrete specimens subjected to conditions that lead to an accelerated progression of alkali-silica reaction were developed under the LWRS program, as documented in [11]. This is a reasonable beginning to such an effort, but a much more comprehensive set of simulations of experimental tests as well as simulations of representative structures is needed to bring this capability to the point where it can be confidently deployed. 


\section{Chapter 5}

\section{Multiscale-Informed Modeling of High-Temperature Component Response with Uncertainty Quantification}

\subsection{Introduction}

One of the major issues of concern for advanced reactor structural components is creep, which is a much larger concern at the elevated temperatures for those applications than it is for LWR applications. A joint effort between Argonne National Laboratory, Idaho National Laboratory, and Los Alamos National Laboratory is underway to develop and deploy constitutive models targeted at predicting the life of Grade 91 alloy components subjected to high-temperature environments typical of those that structural components in advanced nuclear reactors would experience. This work is described in detail in [21, 22], but a brief summary of this work is provided here because this was an important part of INL's structural material simulation work in FY 2020.

\subsection{Creep Material Models}

To address high-temperature creep in advanced reactor metals, two fundamentally different types of material constitutive models have been integrated into Grizzly. The first approach uses traditional phenomenological models, the parameters of which are calibrated to match experimental observations of the response of a material of interest in a given regime. These models are provided through the Nuclear Engineering Material model Library (NEML), which is an open-source library of common classical material models that can be combined in a variety of ways. This library, developed at Argonne National Laboratory, can optionally be linked with Grizzly. Parameters for the NEML Grade 91 model have been calibrated based on experimental data for a wide range of Grade 91 alloys that have undergone a variety of processing. A Bayesian approach was used to derive distributions of uncertain parameters for this model based on this data set.

The second class of material models explored here are reduced order models (ROMs) that are derived from mining a synthetic database of predicted mechanical response derived from the use of a crystal plasticity based mechanistic constitutive model. The visco-plastic self-consistent (VPSC) polycrystal plasticity model was used here to compute the response of a representative volume of material under mechanical loading and account for the effects of microstructural features. Directly evaluating a mesoscale model at every material point in an engineering-scale model is possible but cost-prohibitive. To provide the benefits of a mesoscale-based model that is aware of microstructure, a mesoscale model is evaluated under a wide range 
of conditions of interest, and data analytics are used to develop a ROM that represents the material response using functions that are readily evaluable within an engineering constitutive model. A set of such models, known as LAROMANCE, for a growing number of alloy types has been developed by Los Alamos National Laboratory.

\subsection{Simulation Results}

The NEML and LAROMANCE Grade 91 material models have been integrated in the Grizzly code, which was used to perform proof-of-concept uncertainty quantification analyses of simple components under prototypical conditions [21]. In this work, we simulated the cross-section of a pipe and a slightly more complicated 2D axisymmetric sphere nozzle model shown in Figure 5.1(a). The built-in stochastic analysis capabilities in the MOOSE framework that Grizzly is built on are used to run large sets of simulations for this uncertainty quantification analysis of input material properties. Simulations were run for 20 years or until the maximum principal strain reached a critical level. Results for the sphere nozzle geometry using the LAROMANCE Grade 91 material model are shown in Figure 5.1(b). These distributions are used to correlate critical levels of principal strain with material input properties. For the results in Figure 5.1(b), we observed that a high initial precipitate content in the LAROMANCE Grade 91 model increased the creep rate at higher temperatures. We also observed that the LAROMANCE and NEML models resulted in very different creep responses due to the type of data to which they are fit. These simulations also demonstrated that a reduced-order modeling approach can be successfully deployed to propagate uncertainties from the material scale to practical engineering-scale component simulations.

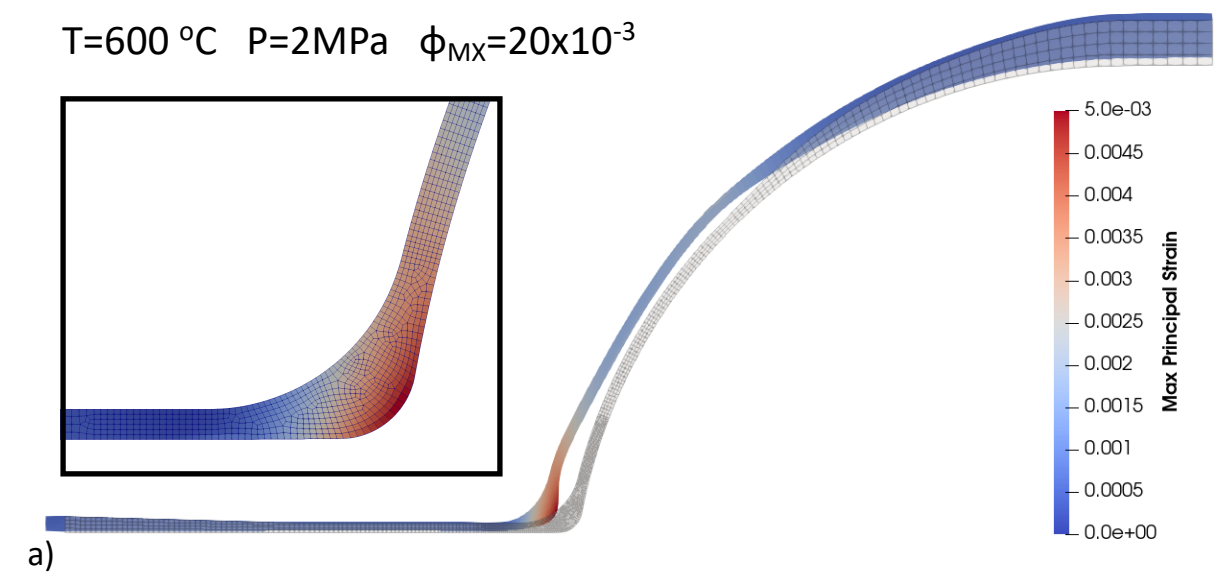

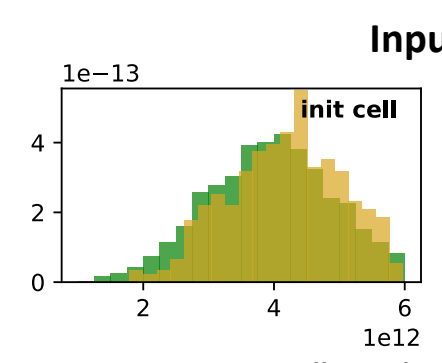

b)

Input Parameters ( $\mathrm{T}=600 \mathrm{C}, \mathrm{P}=\mathbf{2 M P a})$
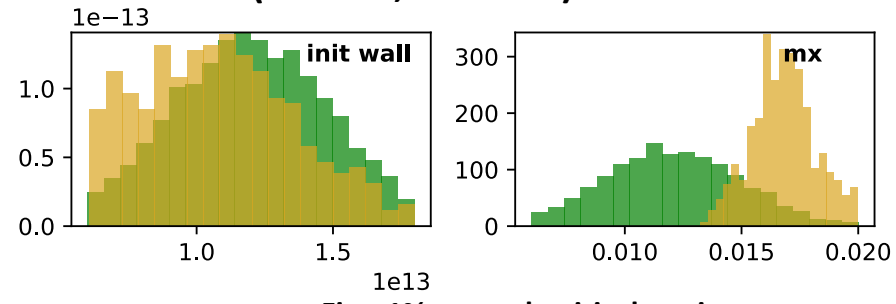

First $1 \%$ to reach critical strain

Figure 5.1: (a) Constant pressure simulation results at $2 \mathrm{MPa}$ and $600^{\circ} \mathrm{C}$ for LAROMANCE Grade 91 with a high MX phase content. Final maximum principal strain contours on a $20 \times$ displaced mesh, compared to the undeformed mesh in gray. (b) Normalized histograms of input material parameters for 4,800 samples are shown in green and the earliest $1 \%$ of simulations to reach the critical strain are shown in gold. 


\subsection{Future Work}

Details of logical follow-on work are discussed in [21]. These tasks are summarized at a high level here:

- The NEML Grade 91 material model requires improvements for cyclic loading. These improvements should include an improved representation of kinematic hardening in the plasticity models for cyclic loading. The Bayesian MCMC method should also be improved to better fit experimental scatter.

- The LAROMANCE Grade 91 material models will provide a better estimate of the structure's variability if the component geometry is stochastically seeded with randomized material properties.

- The LAROMANCE model does not yet include tertiary creep and damage. By basing the material response on a spatially resolved microstructure model that includes these effects, this model can be expanded to include those effects.

- This work has demonstrated the applicability of the LAROMANCE model under relatively narrow conditions. The robustness of this constitutive modeling approach under a broader range of conditions can be improved with further development.

- Further development of the stochastic sampling tools used in Grizzly, including the use of more sophisticated adaptive importance sampling, will permit accurate sampling in the tails of the distributions with lower computational resource demands.

- The engineering scale analysis tools need further development to include the effects of damage.

- More realism is needed in the engineering-scale component models and in the failure criteria. This study has demonstrated a reasonable proof of concept, but models that push the constitutive models into more numerically challenging regimes will better demonstrate the robustness of this approach on more representative real-life problems. 


\section{Chapter 6}

\section{Spectral Solver in the MOOSE Environment}

\subsection{Introduction}

Material mechanical behavior, such as creep, fatigue, and fracture, is influenced by the interaction of microstructurelevel components. Grain boundaries and filler-matrix systems exhibit a complex local behavior that determines the macroscopic physical performance of the materials. For these reasons, the ability to model microscopic component interactions is paramount to the design and mechanical behavior prediction of a wide variety of materials. Microstructures are often modeled as a representative volume element (RVE) with periodic boundary conditions. Physical behavior such as diffusion or elasto-plastic response is then resolved in the RVE.

Two modeling approaches are commonly employed to resolve the physics within the RVE. The more conventional and mature finite element method (FEM) uses a Galerkin approach to represent spatial variation of solution fields. On the other hand, spectral solvers were established on the idea of solving a nonhomogeneous Poisson equation with a choice of reference material, that is, the Lippman-Schwinger equation (see [23]), and use the Fast Fourier Transform (FFT) to solve a unit cell problem on a regularly spaced grid. The numerical performance of FFT, which in its discrete form scales by $\mathcal{O}(n \log n)$, allows for a finer problem resolution compared to the FEM, whose scaling depends on the preconditioner [24] and is less performant.

The original FFT solver methodology introduced by Moulinec and Suquet [23] is based on the definition of an auxiliary problem where the material's stiffness was divided into a homogeneous linear elastic stiffness and a polarization field, which depended on the location in the RVE. Expressions for the Green's function can be obtained analytically in the reciprocal space. This approach has been successfully employed to model mechanical behavior of nonlinear composite structures [23] and viscoplastic polycrystals [25]. For these applications, spectral solvers have demonstrated superior performance and fewer numerical problems, as mesh distortion was avoided.

The past decade has seen new developments in the formulation and implementation of FFT solvers. The micromechanical problem can be recast as a variational one that allows for distinguishing various components, such as the discretization, constitutive linearization, and the solution of the nonlinear system, which, in turn, facilitates their individual optimization, see [26]. Indeed, this more general approach makes the auxiliary problem obsolete and replaces it with a mapping onto compatible field variables. This recent approach has been successfully exercised in recent works with an extended focus on numerical optimization and accuracy, see [27, 28].

The MOOSE framework, which is the basis for many of the simulation codes being developed by the NEAMS program, is based on FEM. The software environment of MOOSE offers significant advantages in many areas. However, for the class of RVE simulations that can be addressed using spectral methods, spectral methods offer compelling performance advantages over FEM. The current effort was undertaken to 
explore the feasibility of implementing a spectral solution method based on MOOSE. This could potentially result in significant performance improvements on this important class of problems, while leveraging the advantages that the MOOSE software environment provides, and potentially even allow reuse of some of the software modules currently used in FEM solutions of the same types of problems.

With an eye on the variety of existing spectral solver formulations, this effort focused on the creation of a necessary application programming interface (API) in MOOSE [29] that enables the creation of methodologies and algorithms for general FFT-based solutions. No assumption is made as to the solver technology or the problem that is solved; instead, the initial implementation of the spectral solver allows the application developer to create his or her own algorithms.

\subsection{Implementation}

The API for the solution of spectral solvers has been developed in MAGPIE (Mesoscale Atomistic Glue Program for Integrated Execution). MAGPIE is a MOOSE-based application that draws on the MOOSE framework and module capabilities ${ }^{1}$.

A number of classes were added to support the new spectral solve type, which has various characteristics:

1. Variables are represented as a buffer of real and complex numbers using the standard library's C++ std: : vector

2. No assumption is made as to the solution methodology

3. The developer can easily perform forward and backward (inverse) Fourier transforms by simply calling a method on the buffer

4. The underlying Discrete Fourier Transform (DFT) solver used here is FFTW ${ }^{2}$. The buffer implementation allows for the use of other DFT back ends.

The developer can then create new algorithms for the solution of a variety of problems, including heat diffusion, nonlinear elasticity, and viscoplastic behavior of polycrystals. More specifically, the implementation of the spectral solver API involves the following $\mathrm{C}++$ classes:

- FFTData creates the objects that contain buffers of data on which the developer may perform basic mathematical operations. This class is templated to allow for real, vector, and various tensor-valued entities. FFTData contains a variety of operators (e.g. addition, subtraction, multiplication, division, power, and a number of helper functions that allow usual operations in the development of mechanical material models, such as the computation of deviatoric stresses or volumetric pressure).

- FFTProblem. This class features many capabilities for solving nonlinear equations plus auxiliary equations, which are inherited from MOOSE's FEProblem. FFTProblem adds the possibility of operating on buffers of FFT variables, thereby allowing the implementation of spectral solvers.

- FFTBufferAux. The developed API adds an auxiliary kernel to assign initial conditions to the variables.

- FFTWBufferBase allows to call FFTW to perform discrete Fourier transforms through real-to-complex (r2c) fftw_plans. FFTWBufferBase inherits from FFTBufferBase and holds references to FFTData. In addition, it has data members describing the grid.

\footnotetext{
${ }^{1}$ see repository https://github.com/idaholab/magpie

${ }^{2}$ http://www.fftw.org/
} 
- SpectralExecutionerBase. This is an ad hoc executioner that holds a reference to an FFTProblem and initializes and executes the problem's variables and parameters.

- SpectralExecutionerDerived. This class inherits from SpectralExecutionerBase and provides the components to develop FFT-based solutions to various physical problems (e.g. the diffusion equation and nonlinear plasticity).

To solve a new physics problem, the application developer needs to inherit from SpectralExecutionerBase and override the methods that initialize and execute the problem variables. As an example, we show below a verbatim excerpt of the SpectralExecutionerDiffusion: : execute() method that implements a simple diffusion problem where the solution advances with a given time step _dt. The function getGreensFunction computes the corresponding Green's function of the diffusivity problem in the real space. The buffer methods forwardRaw () and backward () carry out direct and inverse discrete Fourier transforms, to the Fourier and real space, respectively. These classes provide basic mathematical operators that can be applied to the buffers, for example, u_buffer.reciprocalSpace () *= greens.reciprocalspace().

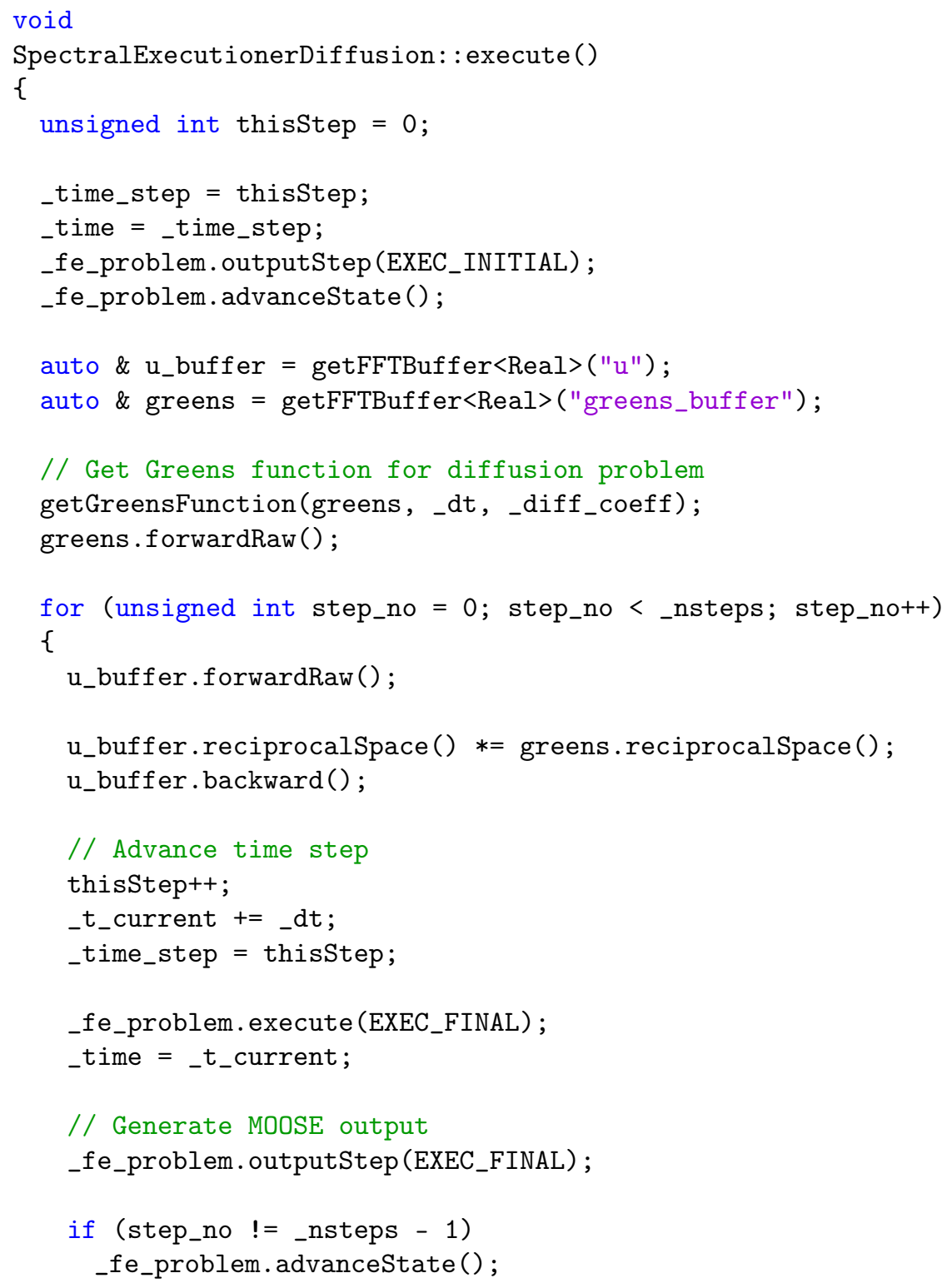


In addition to a two-dimensional diffusion solver, a linear elastic material executioner for a single loading step was created, SpectralExecutionerLinearElastic. At present, the aforementioned implementations work serially and rely on the application developer to provide the algorithm. Initial verification of FFT spatial derivatives, the diffusion equation solver, and a linear elastic material solver are presented in the numerical results section 6.3 .

\subsection{Numerical Results}

This section shows some verification results obtained using the spectral solver's API in MAGPIE.

\subsubsection{Numerical differentiation verification}

Spatial derivatives may be taken by differentiating FEM basis functions or by direct point-wise multiplication of the function to be differentiated by its corresponding $k$ vector. In this subsection, we verify that MAGPIE's FFT and FEM spatial derivatives are within the same tolerance. The two-dimensional function used is an ellipse with a semi-axis $a=30$ and $b=15$, the ellipse's internal value is 1.0 , whereas is 0.0 outside the ellipse area. There is a smooth transition between the two values as depicted in Figure 6.1.

The class SpectralExecutionerBase has an execute() method that performs spatial derivatives by point-wise multiplying the function in the reciprocal space by $\frac{2 \pi i}{L} k$, where $i$ is the imaginary unit, $L$ is the domain length in one dimension, and $k$ is an integer referring to summation in the Fourier space. Excellent agreement between the two differentiation methods can be inferred from Figures 6.2 and 6.3.

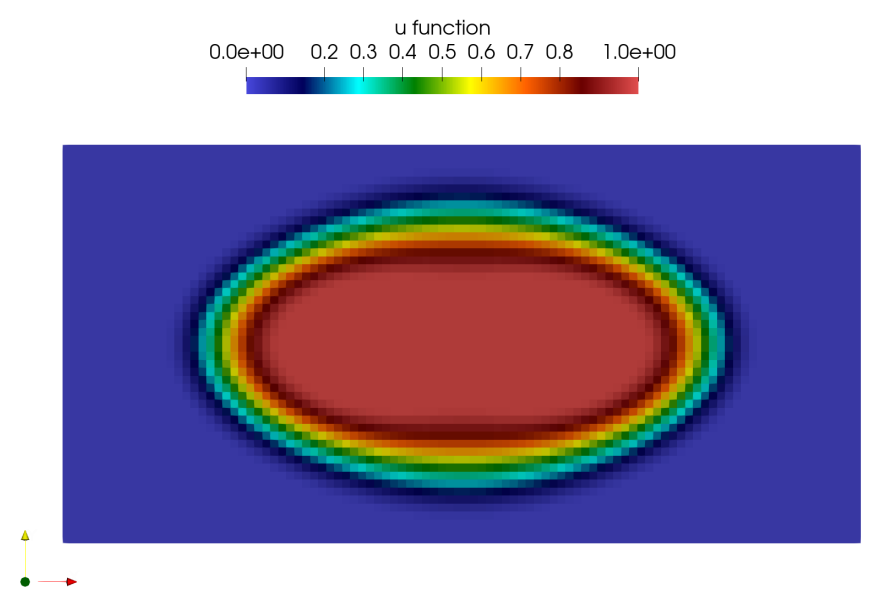

Figure 6.1: Ellipse-like function. 


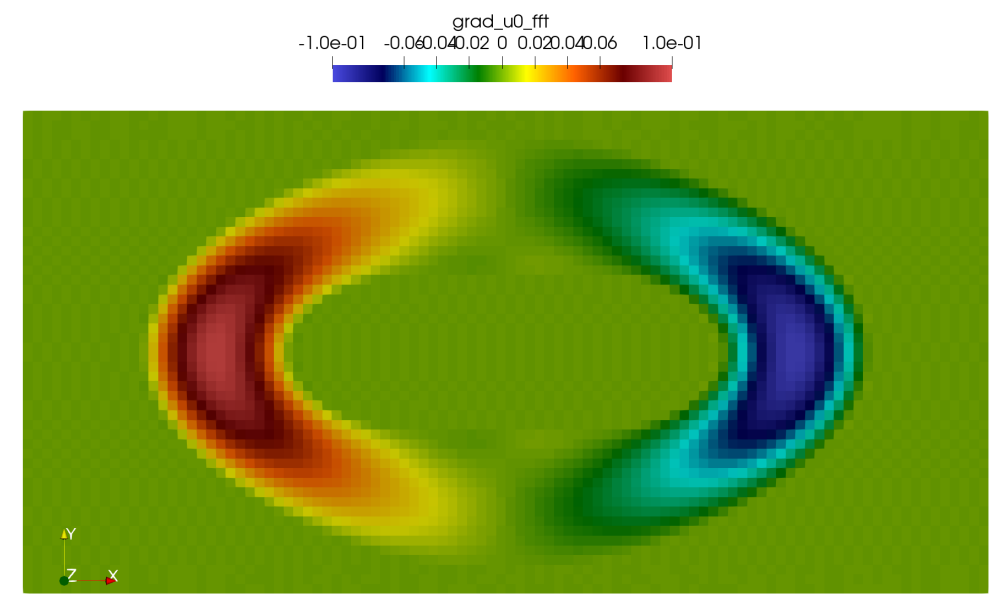

Figure 6.2: FFT-based spatial X derivative.

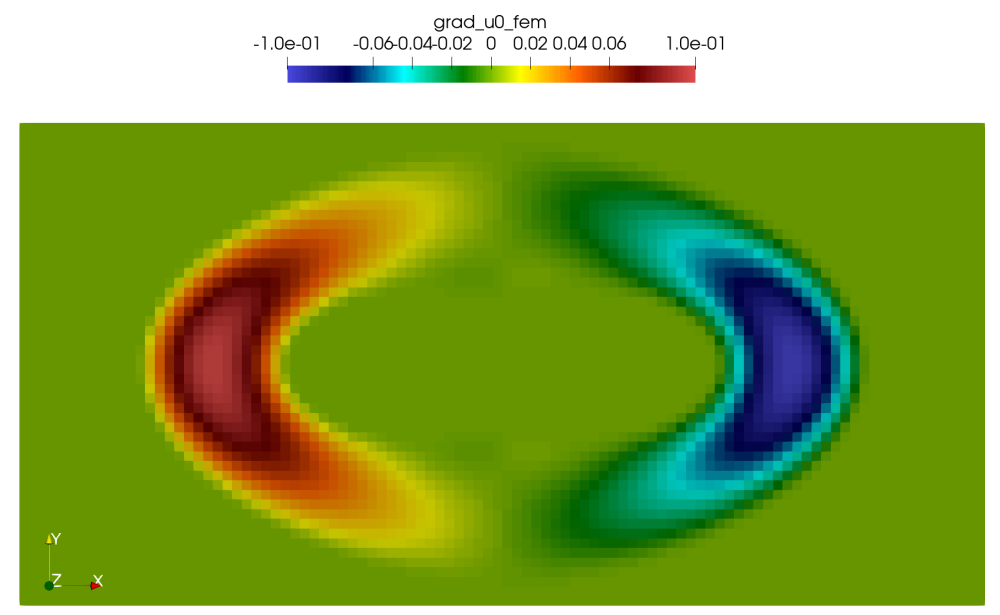

Figure 6.3: FEM-based spatial X derivative. 


\subsubsection{Solver verification - Diffusion problem}

The spectral solver implementation outlined in Section 6.2 is verified here against MOOSE's finite element capabilities. The problem studied here has 100 by 100 dimension units and two-dimensional grids/meshes of 20 by 20,200 by 200, and 2,000 by 2,000. The simulation time is $0.1 \mathrm{~s}$, with a diffusion coefficient of 400 and a time step of $5 \mathrm{~ms}$. The initial value of the diffused variable is 1 within a circle of radius 1 and 0.0001 everywhere else, see Figure 6.4.

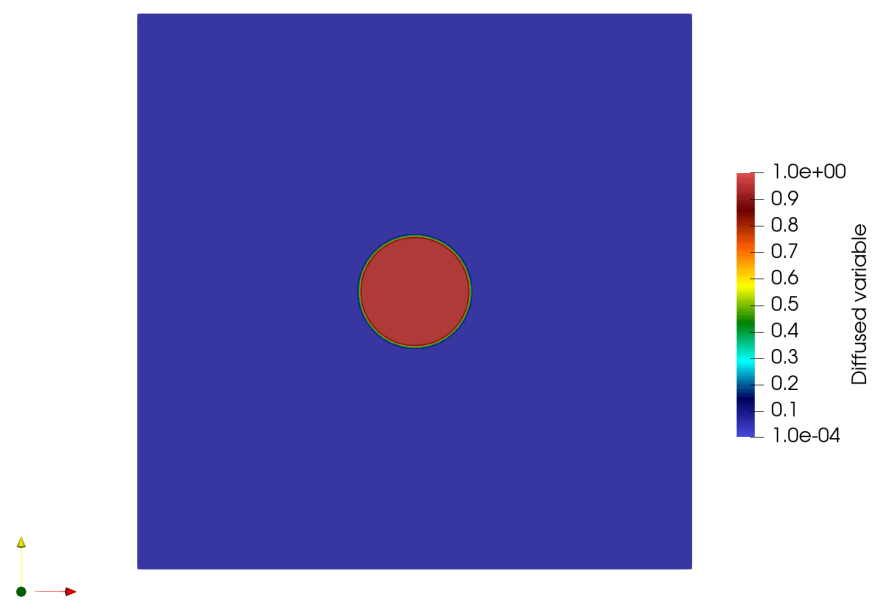

Figure 6.4: Initial conditions for the diffusion problem.

Figures 6.5 through 6.7 show a comparison of the FEM and the spectral solver for the diffusion simulation outlined above for a 200 by 200 grid. Despite the FEM and the spectral solver solving the problem differently, local basis functions vs a global approach, the agreement is good as long as a sufficient number of cells/elements are used. 


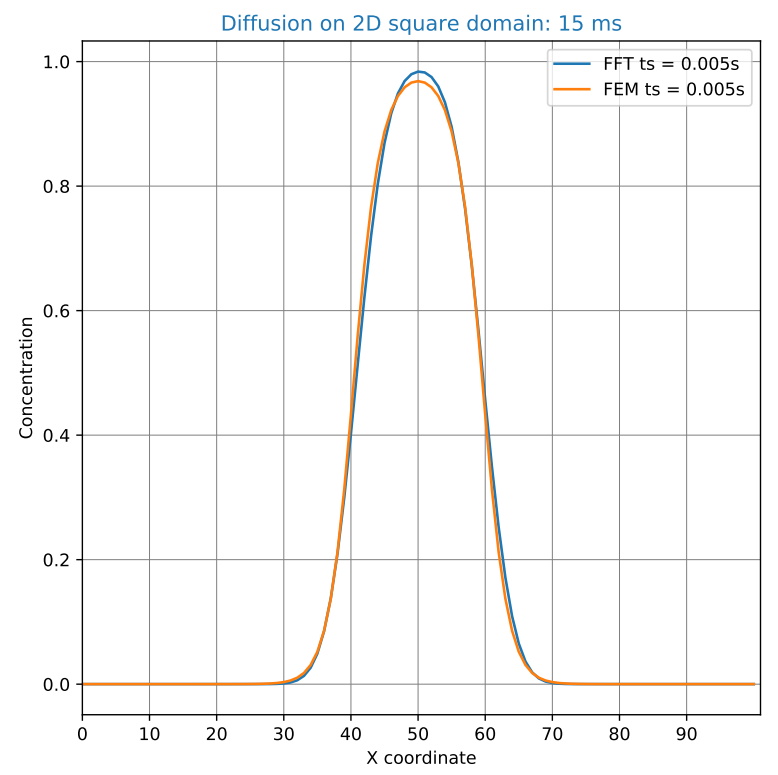

Figure 6.5: Diffusion variable at $t=0.015 \mathrm{~s}$. Time step $t_{s}=0.005 \mathrm{~s}$, solution on a two-dimensional 200 by 200 grid.

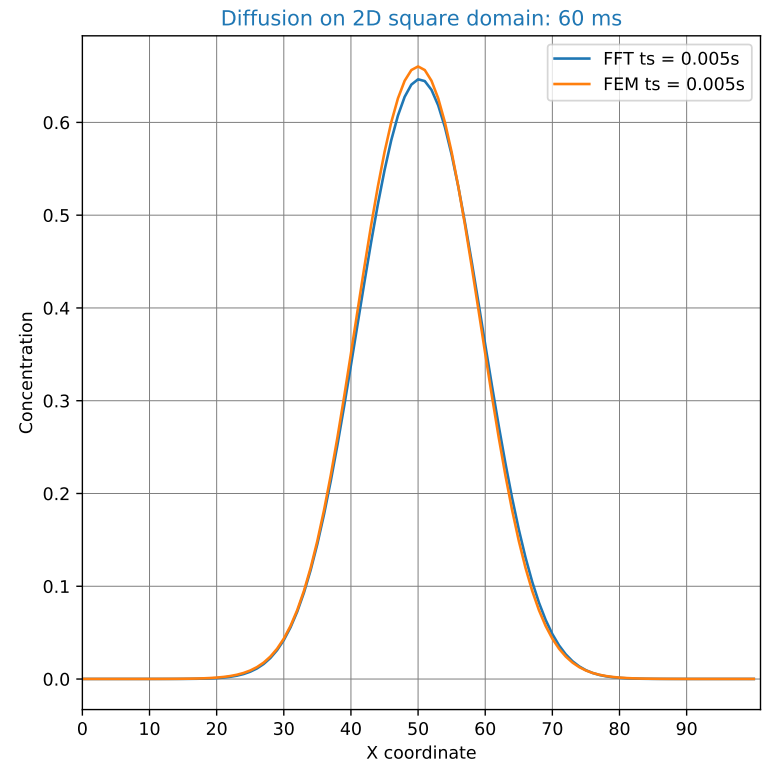

Figure 6.6: Diffusion variable at $t=0.060 \mathrm{~s}$. Time step $t_{s}=0.005 \mathrm{~s}$, solution on a two-dimensional 200 by 200 grid. 


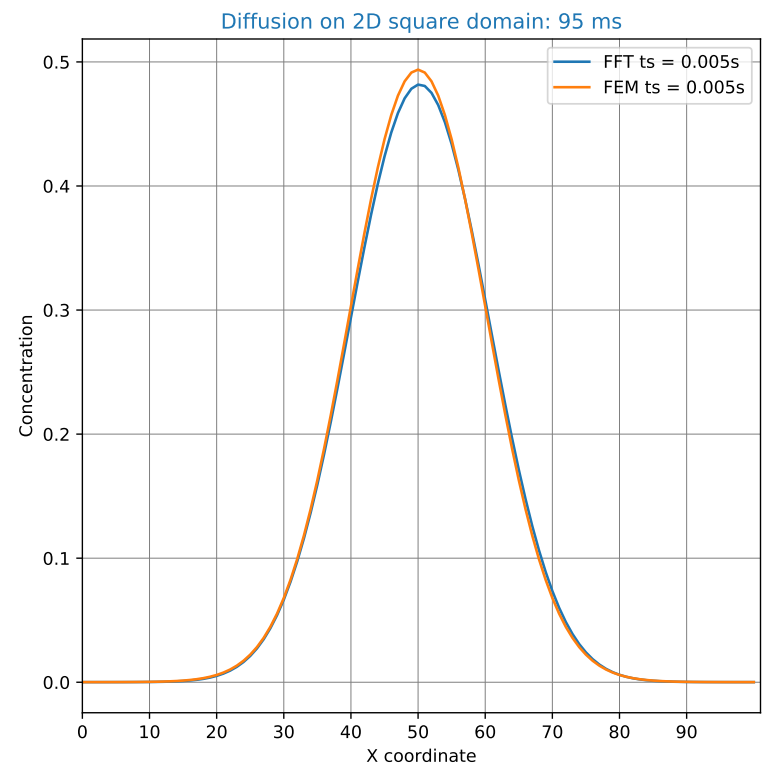

Figure 6.7: Diffusion variable at $t=0.095 \mathrm{~s}$. Time step $t_{s}=0.005 \mathrm{~s}$, solution on a two-dimensional 200 by 200 grid.

\subsubsection{Solver verification - Linear elastic problem}

A linear elastic spectral solver was developed and verified against a finite element model. The algorithm used in this work is outlined in Algorithm 1. The algorithm is based on the original work in [23], where a reference medium captures an average of the materials composing the solid. Here, the straightforward choice $\lambda_{0}=\max (\lambda(\mathbf{x})) / 2.0$ and $\mu_{0}=\max (\mu(\mathbf{x})) / 2.0$ is made, where $\lambda$ and $\mu$ are the modulus of elasticity and the shear modulus, respectively. A Green's operator in the Fourier space, $\hat{\boldsymbol{\Gamma}}_{0}(\boldsymbol{\xi})$, may be built with these average medium properties, which is used to iterate on the strains.

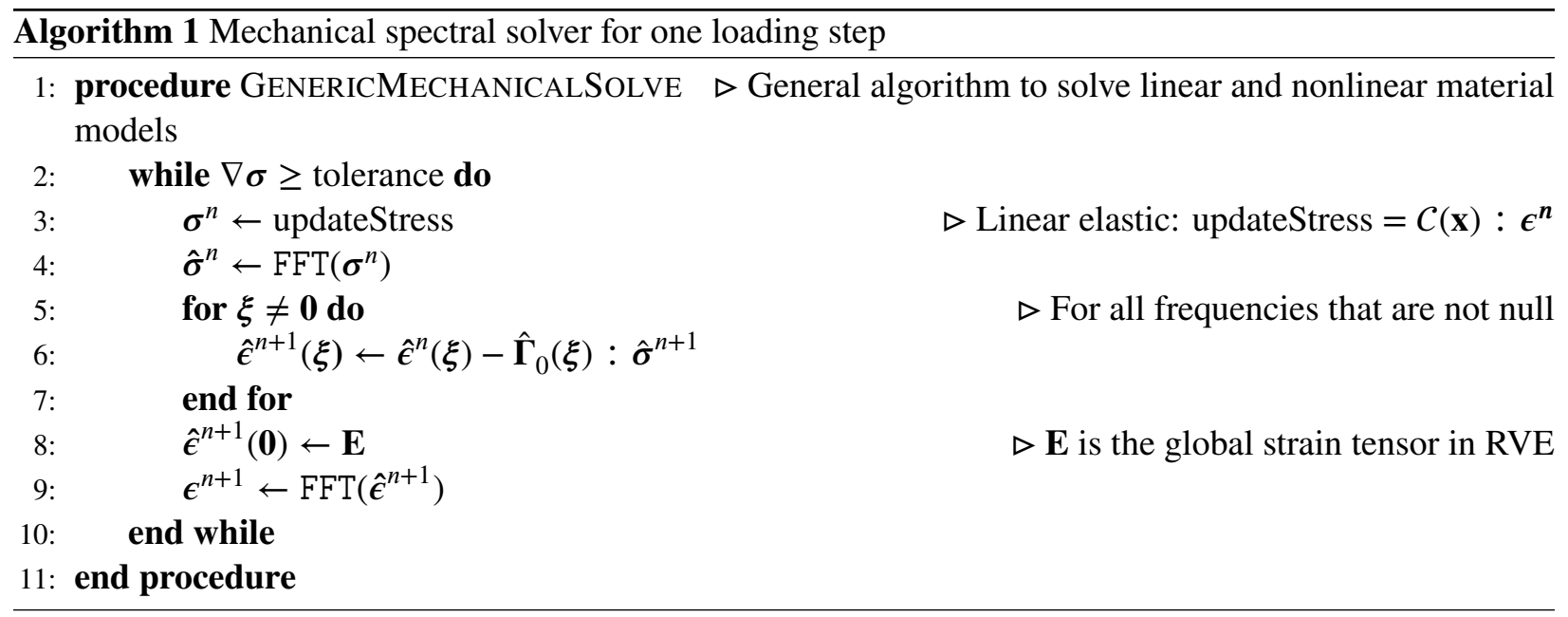

The verification of the linear elastic implementation is performed on a three-dimensional RVE with two 
phases. A central sphere of radius 2.0 is made up of a linear material with a modulus of elasticity of $1.0 \times 10^{4}$ and a Poisson ratio of 0.3 ; the rest of the $10 \times 10 \times 10 \mathrm{RVE}$ is composed of a linear elastic material four times stiffer. The global strain $\mathbf{E}$ imposed is $\left(\epsilon_{y z}, \epsilon_{x z}, \epsilon_{x y}\right)=\left(4.39 \times 10^{-3},-2.57 \times 10^{-2}, 1.00 \times 10^{-2}\right)$. Figures 6.8 and 6.9 show $y z$ strain and stress spatial variation, respectively, for various mesh and grid sizes. It is observed that the basic spectral algorithm implemented causes spatial oscillations in the results for smaller grids. For finer grids (e.g., $256 \times 256 \times 256$ ), the results are smoother and better fit the RVE's phases. Agreement between FEM and FFT results is good. The red lines in Figures 6.8 and 6.9 show the results when the tolerance on the resulting stress is tightened to $10^{-9}$; however, differences are barely noticeable compared to the default tolerance of $10^{-5}$ on the global equilibrium used by default throughout this work.

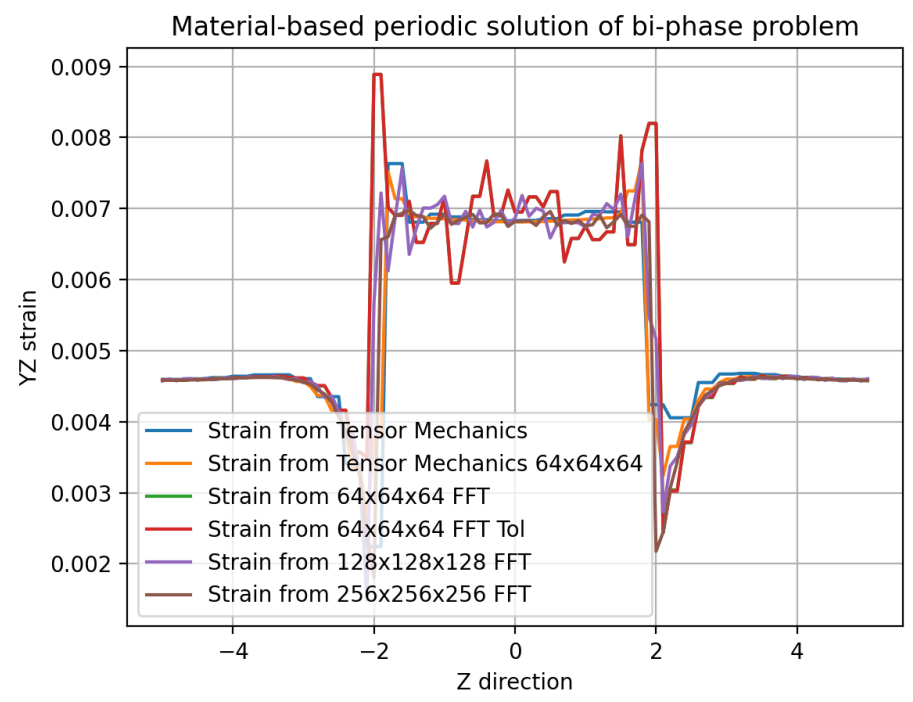

Figure 6.8: Spatial variation in $y z$ shear strain for various grid/mesh sizes along the $z$ direction: Comparison between FEM (Tensor Mechanics) and FFT results.

\subsubsection{Computational efficiency}

We present here a brief summary of the computational performance of the two-dimensional diffusion problem in MOOSE. The linear diffusion problem is solved by a general nonlinear approach with nonlinear and linear iterations (i.e. the FEM solver) and by using the new spectral solver capability.

Table 6.1: FEM vs FFT: CPU times for a two-dimensional diffusion problem.

\begin{tabular}{|c|c|c|c|}
\hline \multicolumn{4}{|c|}{ CPU time } \\
\hline Number of cells & Finite element method & Spectral solver & Speedup \\
\hline $4 \times^{2}$ & $0.578 \mathrm{~s}$ & $0.127 \mathrm{~s}$ & 4.55 \\
$4 \times^{4}$ & $48.16 \mathrm{~s}$ & $2.679 \mathrm{~s}$ & 17.98 \\
$4 \times^{6}$ & $38,702 \mathrm{~s}$ & $239.90 \mathrm{~s}$ & 161.3 \\
\hline
\end{tabular}

Table 6.1 lists CPU times and speedup estimates for various runs using FEM and FFT approaches. It is apparent that the spectral solver performs differentially better for this problem even as the number of 


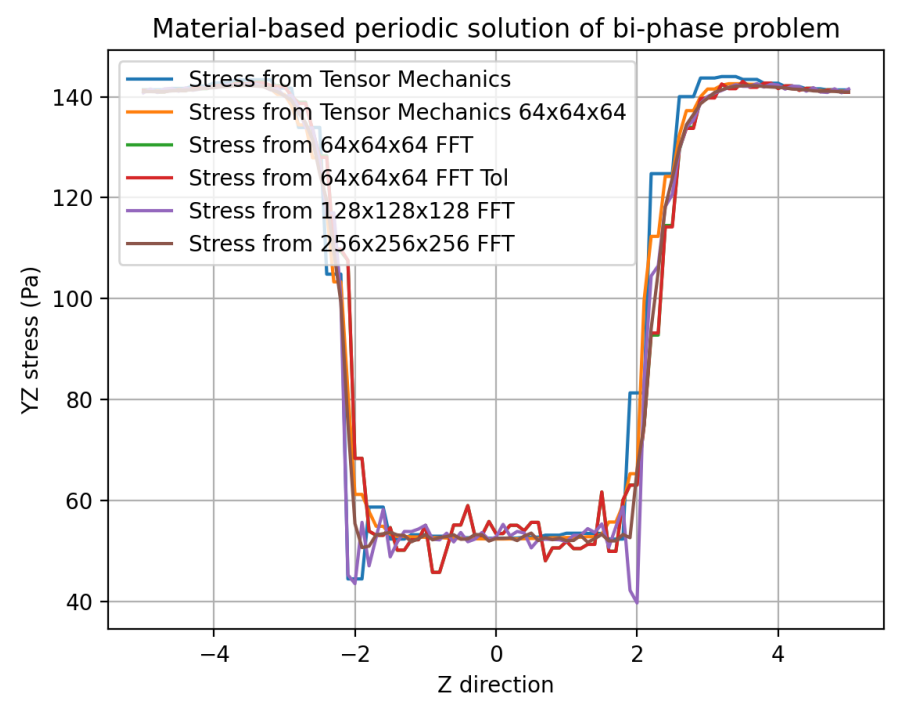

Figure 6.9: Spatial variation of $y z$ shear stress for various grid/mesh sizes along the $z$ direction: Comparison between FEM (Tensor Mechanics) and FFT results.

pixels/quads increases. In addition, Figure 6.10 shows basic profiling information for a spectral run with four million two-dimensional cells.

The results in 6.1 can be qualified as follows. The number of iterations required to solve the FFT problem is barely increased by increasing the grid sizes by an order of magnitude. However, increasing the number of finite elements (quads) lowers the preconditioner effectiveness and significantly increases the number of linear iterations. For example, going from an FEM mesh of 200 by 200 elements to a mesh of 2,000 by 2,000 elements puts an additional burden on the linear solves, which average 18 iterations in the former case and 250 iterations in the latter. This side effect is not present in the spectral solver.

The elastic solutions presented in Section 6.3.3 were obtained from three-dimensional bi-phase elastic materials. Comparing a 64 by 64 by 64 first-order finite element mesh with a 256 by 256 by 256 FFT cell grid, we observe that a finite element solver computes a 12.9 integration or Gauss points per second, and the spectral solver computes 7,294 cells per second (i.e., a speed up of more than 500). Despite the theory behind both methods being different - the spectral solver uses here a collocation method whereas finite element uses a Galerkin approach - it is apparent that the methodology based on the fast Fourier transform is, at least, two orders of magnitude faster for large problems. This advantage is likely to continue to hold for other mechanical problems.

\begin{tabular}{|c|c|c|c|c|c|c|c|c|c|c|c|c|c|}
\hline । & Section & | Calls | & Self (s) & । & $\operatorname{Avg}(s)$ & $\%$ & | Children(s) & $\operatorname{Avg}(s)$ & $\div$ & I & Total (s) & 1 & $\operatorname{Avg}(s)$ \\
\hline & MagpieApp (main) & 11 & 0.012 & । & 0.012 । & 0.00 & 239.900 & 239.900 & 100.00 & । & 239.912 & 1 & 239.912 \\
\hline । & FFTProblem: : computeUserobjects & 11 & 3.786 & i & 3.786 & 1.58 & 0.000 & 0.000 & 0.00 & i & 3.786 & i & 3.786 \\
\hline । & FFTProblem: :computeUserobjects & 201 & 4.706 & 1 & 0.235 & 1.96 & 0.000 & 0.000 & 0.00 & । & 4.706 & 1 & 0.235 \\
\hline । & FFTProblem: :outputStep & $21 \mid$ & 0.141 & i & 0.007 & 0.06 & 56.000 & 2.667 & 23.34 & i & 56.140 & i & 2.673 \\
\hline । & AuxiliarySystem: : computeElementalvars & 201 & 85.277 & i & $4.264 \mid$ & 35.55 & 0.000 & 0.000 & 0.00 & i & 85.277 & 1 & 4.264 \\
\hline
\end{tabular}

Figure 6.10: Performance graph for a diffusion spectral solve in a 2,000 by 2,000 grid.

The examples presented in this document are publicly available in the MAGPIE repository. 


\subsection{Future Work}

The work performed here demonstrates the feasibility of an FFT solver in the MOOSE framework. The newly developed API benefits from many of the existing framework capabilities, such as general parsing, the nonlinear problem, auxiliary variables, kernels, and output files. In addition, MOOSE's spectral solver implementation has the potential to leverage existing material models and equation solvers.

To make the spectral solver usable for practical applications, a number of issues will need to be addressed. So far, diffusion and linear elastic solvers have been implemented and verified. A number of new executioners will need to be developed to include material models of interest to nuclear engineering applications. These models include finite strain formulations and viscoplastic deformation of crystals. These executioners will need to include nonlinear solver strategies to ensure efficient implicit solutions.

Efficient parallelization of the code will enable the solution of more detailed three-dimensional problems. The underlying FFT solver used, the FFTW library, features message-passing interface (MPI) methods that can be called from the spectral solver. This will require changes to MOOSE's API to allow for distributedmemory parallelization and an appropriate domain decomposition within the framework.

The fixed-point iteration solver implemented in MAGPIE can be enhanced by using techniques to accelerate convergence, which may involve the use of conjugate gradient methods. This addition would further enhance the speed of the spectral solver, especially when the material phases have high-constrast stiffnesses.

Finally, end users often need to create a large number of command blocks in the input files. To lower the user's entry point, we envision the creation of MOOSE Actions that would provide shortcuts for most of the tedious input currently required in the input file. Auxiliary variables, kernels, and solve types can be defined within an Action that spawns the creation of the necessary objects.

We believe that with this additional development, an efficient microstructure modeling capability could be provided in MOOSE, which would provide many advantages, including a modern, open, and modular software environment that employs rigorous software quality practices. 


\section{Chapter 7}

\section{D Non-Planar Crack Propagation with the Extended Finite Element Method}

\subsection{Introduction}

The extended finite element method (XFEM) is a technique that allows for the representation of arbitrary discontinuities within an otherwise continuous finite element solution. Historically, representing such discontinuities has required modifying meshes so that element boundaries are positioned at the locations of these discontinuities, which can result in considerable complexity. XFEM allows these discontinuities to pass arbitrarily through the finite-element mesh, which offers significant benefits for modeling discontinuities that evolve over time, such as those that arise from fracture or moving material interfaces. XFEM is made available to all MOOSE-based applications through the "XFEM" MOOSE module. XFEM development in MOOSE was originally motivated by the need to simulate fracture in nuclear fuel [30], but this has also been applied extensively to simulate fracture in structural materials applications in the Grizzly code. One particularly powerful aspect the MOOSE implementation of XFEM is that it can be used to represent discontinuities in any solution field in a multiphysics simulation, which opens up a large number of novel applications.

The present work documented here enhances the XFEM module of MOOSE to enable the modeling of (1) arbitrary non-planar 3D crack growth and (2) subcritical crack growth. These are both important capabilities for modeling fracture in nuclear power plant structural components that were previously lacking. The present work included the benchmarking of the domain integral calculation for non-planar cracks, computation of the direction and velocity of crack extension at discrete points of the crack front, and growing cracks by using the front matching algorithm. The enhanced XFEM module will be useful for high-fidelity modeling of the 3D cracking failure of nuclear structure components in both critical and subcritical conditions, although it also has numerous other potential industrial applications.

\subsection{Non-Planar Crack Propagation}

The non-planar crack propagation capability builds upon the previously developed planar crack propagation capability [31], which involves coupling of a "mesh cutter" class, which defines the location of a discontinuity, with fracture integral calculations. Multiple types of mesh cutters are allowed in MOOSE, including models based on plane geometries or level set functions. The mesh cutters used here define a cutting plane using a mesh of the crack defined using 2D triangles in a 3D space. This approach lends itself well to defining complex crack paths in models that represent the domain using 3D continuum finite elements. The upgrades for new capabilities required several changes to the code. A numerical example will be introduced first to 
facilitate the discussion of these changes.

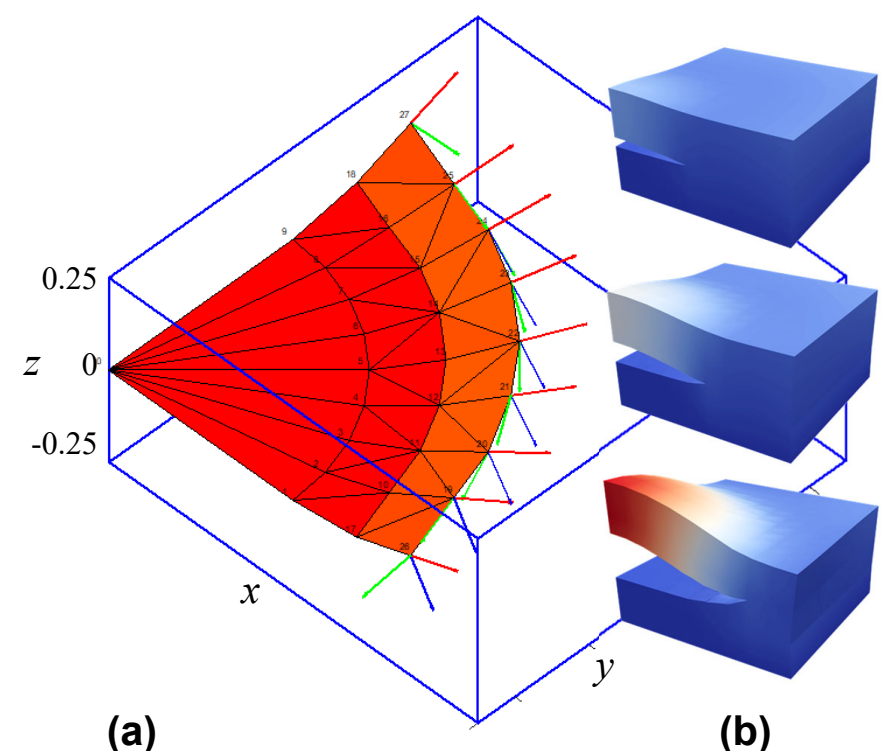

(a) (b)
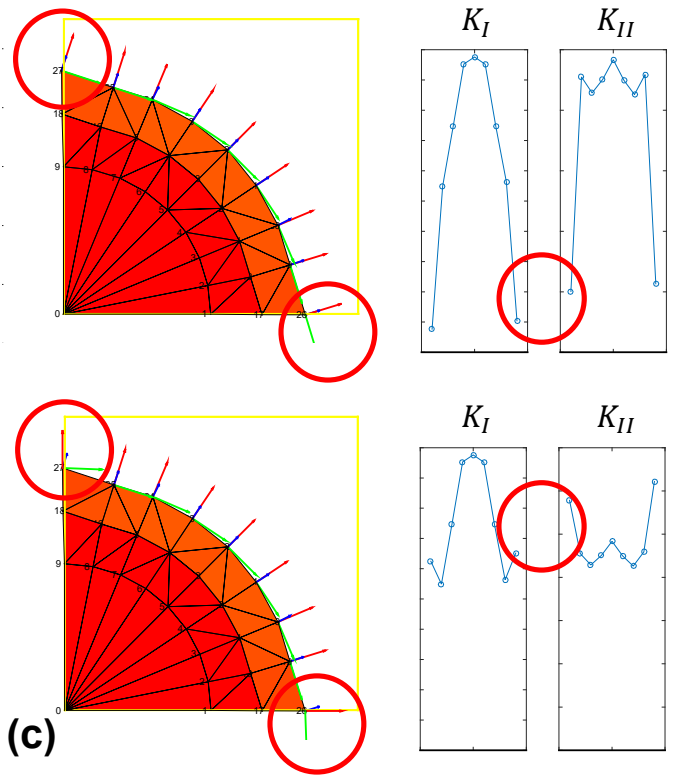

Figure 7.1: Non-planar crack propagation: (a) example with a growing penny-shaped crack; (b) deformed structure as the crack grows; and (c) importance of end nodes treatment (above: without treatment; below: with treatment).

Figure 7.1(a) illustrates a quarter model of a penny-shaped crack embedded in a $1 \times 1 \times 0.5$ block. The crack initially has a radius of 0.5 . The top surface of the block is subjected to uniform tension, and the bottom surface is fully constrained. Symmetric boundary conditions are applied on both planes of symmetry at $x=0$ and $y=0$. Due to the asymmetric loading about the crack plane at $z=0$, the crack will curve, leading to a non-planar crack. Figure 7.1(a) shows the crack configuration after two propagation steps. At every discrete crack front point, a local coordinate system is defined by the crack direction $\left(\overrightarrow{v_{c}}\right.$, red arrow), tangent direction $\left(\vec{v}_{t}\right.$, green arrow), and normal direction $\left(\vec{v}_{n}\right.$, blue arrow). Figure 7.1(b) shows three snapshots of the deformed structure as the crack grows. Several changes were made to enable the simulation of such a non-planar cracking problem:

(1) After each step of XFEM simulation, stress intensity factors are evaluated at all active front points, giving $K_{I}$ and $K_{I I}$. The crack extension direction is calculated by applying the maximum hoop stress criterion (i.e. $\theta=2 \tan ^{-1}\left[\left(K_{I}-\sqrt{\left.K_{I}^{2} K_{I I}^{2}\right) / 4 K_{I I}}\right]\right.$ ) in the local coordinate system defined at the crack front points. The direction is then converted into the global coordinate system to grow the crack.

(2) For a crack described by the mesh cutter, the normal vector $\left(\overrightarrow{v_{n}}\right)$ is evaluated by averaging the normal vectors of all elements connected to a crack front point.

(3) The tangent vector $\left(\vec{v}_{t}\right)$ is corrected after all three vectors are calculated. For a general 3D non-planar crack problem, $\vec{v}_{t}$ is calculated by averaging all front segments connected to a crack front point. $\vec{v}_{t}$ and $\vec{v}_{n}$ calculated in these ways may not be perpendicular to each other exactly, making these two axes of the local coordinate system non-orthogonal. The problem is solved by correcting the tangent vector with $\overrightarrow{v_{t}}=\overrightarrow{v_{n}} \times \vec{v}_{c}$. 
(4) For cracks with a curved front (e.g. the penny-shaped crack) numerical calculation of $\vec{v}_{t}$ using discrete front points may lead to nonphysical tangent vectors at two ends of the front, which may influence $K$ accuracy. The top three subfigures of Figure 7.1(c) demonstrate an example. The crack front should be perpendicular to the block's side surfaces at the two circled intersections, but $\vec{v}_{t}$ is not calculated to be perpendicular. As a result, $K_{I}$ and $K_{I I}$ calculations at these points are incorrect, as shown in the top-right two subfigures. By introducing a new option for the fracture integral that controls how the crack extension direction is computed at the ends of the cracks (end_direction_method = END_CRACK_TANGENT_VECTOR), $\vec{v}_{t}$ at these ends are corrected (bottom-left subfigure), which improves the $K$ predictions, as demonstrated in the bottom-right two subfigures.

Implementing all of these changes enables the non-planar crack propagation capability, which predicts the evolving non-planar crack shown in Figure 7.1(a). The capability will be further demonstrated by using a benchmark problem with an inclined elliptical crack.

\subsection{Subcritical Crack Propagation and Life Prediction}

The subcritical crack propagation capability builds upon the non-planar crack propagation capability and the addition of life prediction models. This implementation applies the Paris law as an example:

$$
\frac{\mathrm{d} a}{\mathrm{~d} N}=C\left(\Delta K_{e q}\right)^{m}
$$

where $d a / d N$ is the crack growth per loading cycle, $\Delta K_{e q}$ is the change of an equivalent stress intensity factor during a fatigue loading cycle, and $C$ and $m$ are material constants. For mixed-mode cracking, there are different definitions of $\Delta K_{e q}$ in the literature [32]. The current implementation adopts the following relationship [33]:

$$
\Delta K_{e q}=\left(\Delta K_{I}^{2}+2 \Delta K_{I I}^{2}\right)^{1 / 2}
$$

It will be straight-forward to expand the life prediction library with other fatigue or stress corrosion cracking models that depend on stress intensity factors. With the subcritical cracking capability implemented, there are two parameters in the input that a user must define for a 3D cracking problem:

(1) growth_speed_method = fatigue: use Paris law to define growth speed growth_speed_method = function: use a Parsed function to define growth speed

(2) growth_dir_method = max_hoop_stress: use the max hoop stress law to define growth direction growth_dir_method = function: use Parsed functions to define growth direction

The use of growth_speed_method $=$ fatigue requires the input of:

(1) max_growth_size: $\Delta a_{\max }$, i.e. the growth length for the crack node that has max $\Delta K_{e q}$

(2) paris_law_c: Parameter $C$

(3) paris_law_m: Parameter $m$

Figure 7.2(a) shows a $1 \times 1 \times 0.2$ plate having an edge crack initially with the length of 0.4 and width of 0.2 . Fatigue crack growth is assumed with $C=1 \times 10^{-13}$ and $m=2.5$. The problem is simulated with two cutter meshes. The coarse mesh, as shown in Figure 7.2(b), advances with a step size of $\Delta a_{\max }=0.1$. The finer mesh as shown in Figure 7.2(c) advances with a step size of $\Delta a_{\max }=0.05$. Note that the step size 

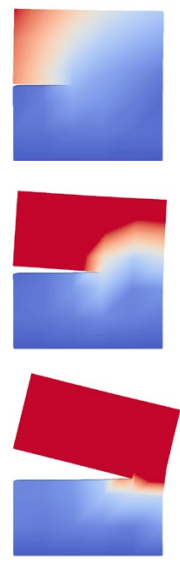

(a)
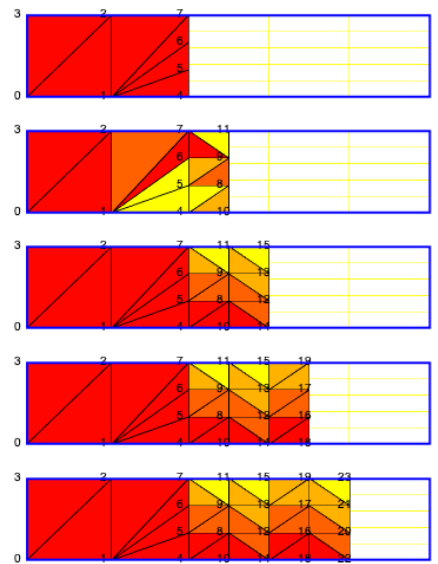

(b)

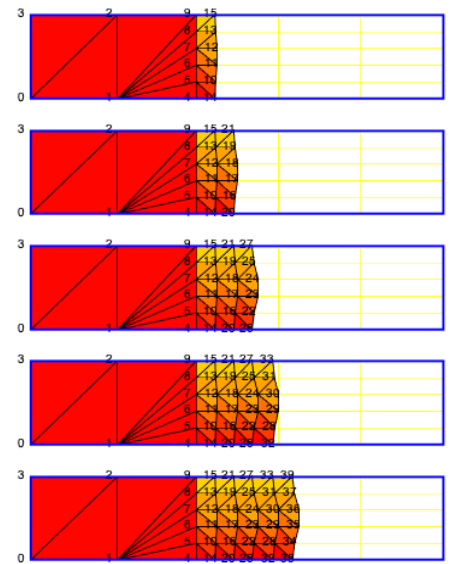

(c)

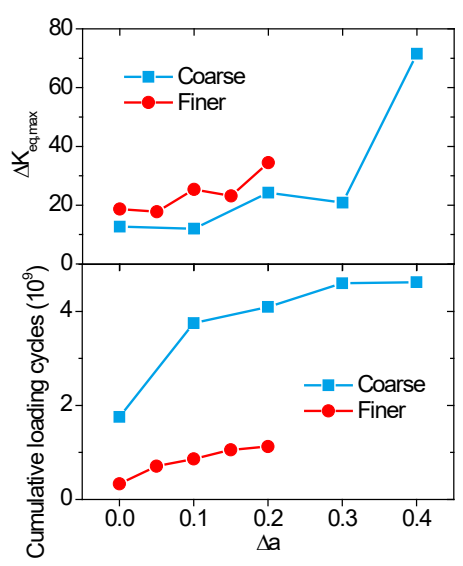

(d)

Figure 7.2: Subcritical crack propagation: (a) an edge crack example; (b) crack propagation described by a coarse cutter mesh; (c) crack propagation described by a finer cutter mesh; and (d) mesh effects on $\Delta K_{e q, \max }$ and cumulative loading cycles.

applies only to the crack front point that has the largest $\Delta K_{e q}$ (i.e., $\Delta K_{e q, \max }$ ). For the other front points, the growth size scales with their respective $\Delta K_{e q}$ following the Paris law (i.e., $\left.\Delta a / \Delta a_{\max }=\left(\Delta K_{e q} / \Delta K_{e q, \max }\right)^{m}\right)$.

Figure 7.2(d) compares $\Delta K_{e q, \max }$ at each simulation step between the coarse and finer meshes. The results show the importance of using appropriate meshes, as the coarse and finer meshes show relatively significant differences in the prediction of $\Delta K_{e q, \max }$. The difference is even more prominent in cumulative loading cycles. It is important to note that, following the Paris law, $\Delta a$ scales with $\left(\Delta K_{e q}\right)^{m}\left(\right.$ e.g. $\left.\left(\Delta K_{e q}\right)^{2.5}\right)$ for the present problem. Any errors in the $K$ predictions will be amplified by the power parameter. It underscores the necessity of accurate $K$ calculations in subcritical crack simulations. The accuracy of the XFEM module will be examined in the next section considering a benchmark with an inclined elliptical crack.

\subsection{Benchmark Simulation: Growth of an Inclined Elliptical Crack}

The enhanced XFEM module is demonstrated here by using an inclined elliptical crack problem that has been considered in the literature as a benchmark $[34,35]$. The problem is challenging for several reasons: (1) the elliptical crack front has a varying curvature; (2) the crack is inclined in a block that is subjected to uniaxial tension, leading to mixed-mode fracture starting from the initial crack; and (3) the initially inclined planar crack will continuously make turns during its growth to maximize the energy release rate, eventually leading to a non-planar crack with relatively complicated geometry. For these three reasons, this problem is particularly suitable to test the ability of a fracture mechanics package to simulate non-planar crack propagation processes.

As shown in Figure 7.3(a), an elliptical crack with the long axis of $a=0.1$ and the short axis of $b=0.05$ is placed at the center (origin) of a large cubic block with an edge length of $h=1$. The ratio of $h / a=10$ is sufficient to safely neglect the boundary effects. The crack is inclined with its surface having an angle of $45^{\circ}$ with respect to the $x-z$ plane. The long axis is along $x$ while the short axis is along $y^{\prime}$. The loading is uniaxial tension with a surface traction of $q=1$ applied on the top and bottom surfaces. Due to the angle of $45^{\circ}$, the crack has a mix-mode nature with non-zero $K_{I}, K_{I I}$ and $K_{I I I}$ at most crack front points. Poisson's 

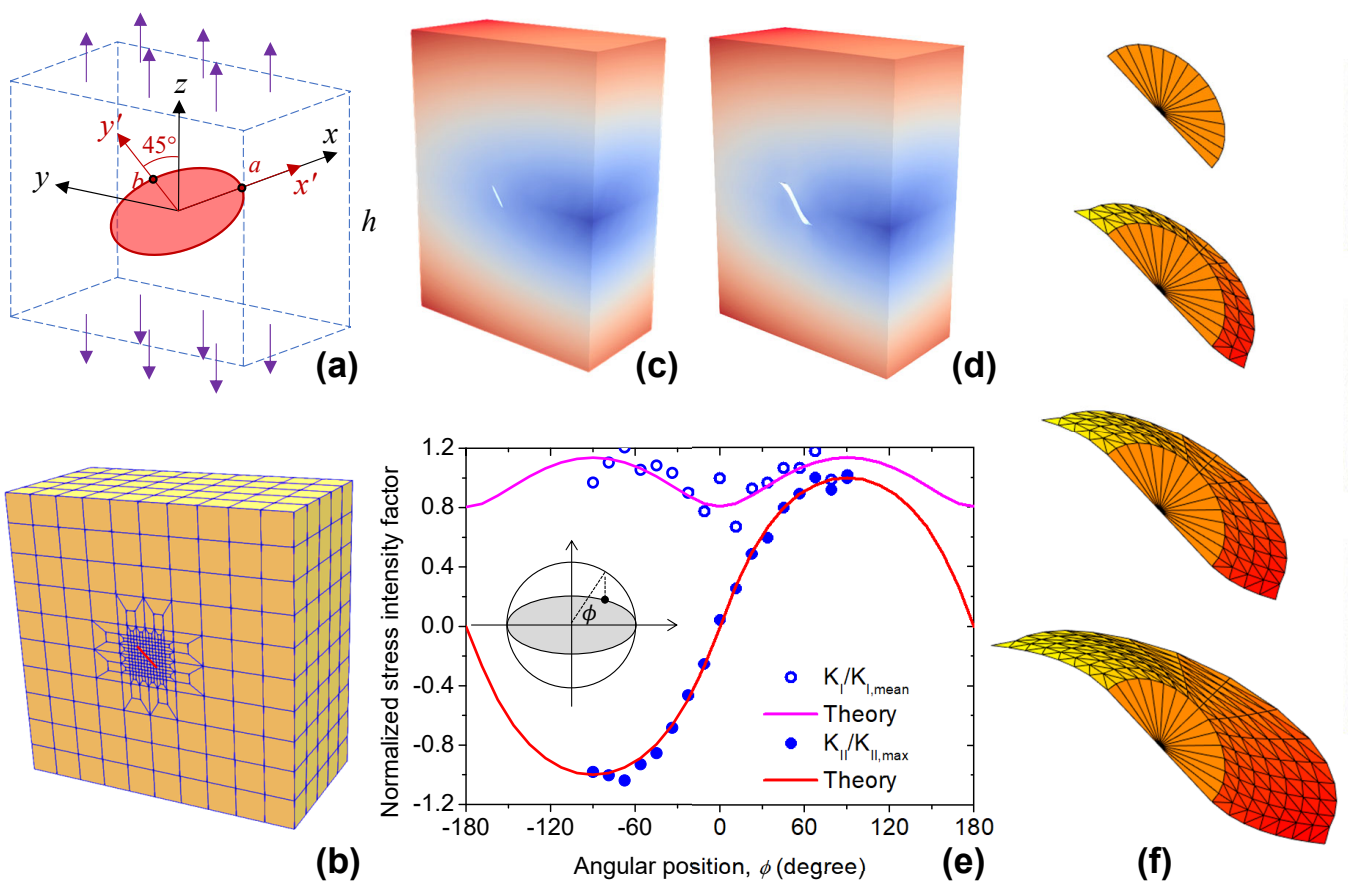

(f)
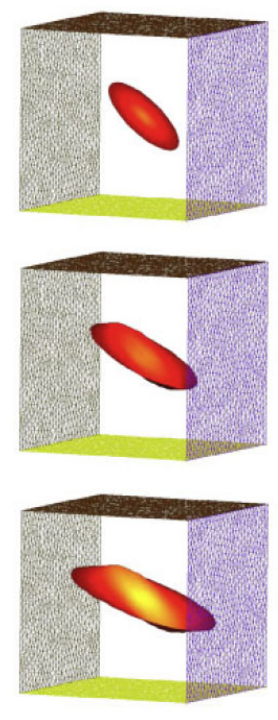

(g)

Figure 7.3: (a) Schematic of an inclined elliptical crack embedded in a cubic block. (b) The finite element model with a red line representing the elliptical crack on the surface. (c) Initial and (d) final crack configurations viewed in the deformed structural model with a relatively large scale factor. (e) Comparison between the numerical and analytical results of two normalized stress intensity factors along the elliptical crack front. (f) Crack surface evolution from a planar elliptical crack to a non-planar crack with complicated geometry.

(g) Crack evolution snapshots in a similar problem (adapted from Figure 14 of Reference [36]).

ratio is $v=0.3$. Young's modulus has no effects on the stress intensity factors.

To improve computational efficiency, only a half of the model $(x>0)$ is analyzed with symmetrical boundary conditions applied on the $x=0$ surface. The model is shown in Figure 7.3(b), where the crack on the surface is represented by a red line. The half model is meshed with 4,130 nodes and 3,764 hex elements. A small region enclosing the inclined elliptical crack is refined to improve accuracy while not significantly compromising efficiency. Figure 7.3(c, d) shows the crack at the initial and final stages, viewed in the deformed structure with a large scale factor of 50,000 for visual clarity.

We first verify the calculation of stress intensity factors as they are critical to determining the crack extension direction as well as the crack growth speed in subcritical problems. The analytical solution of an inclined crack embedded in an infinite domain was determined by Kassir and Sih [37]. The Mode-I stress intensity factor is:

$$
K_{I}=\frac{q}{E(k)}\left(\frac{b}{a}\right)^{1 / 2}\left(a^{2} \sin ^{2} \phi+b^{2} \cos ^{2} \phi\right)^{1 / 4}
$$

where $E(k)$ is the complete elliptic integral of the second kind with $k=\left[1-(b / a)^{2}\right]^{1 / 2}$ and $\phi$ is the angular position of any point on the ellipse, as defined in the inset of Figure 3(e). The Mode-II stress intensity factor is:

$$
K_{I I}=-\frac{1}{(a b)^{3 / 2}}\left(a^{2} \sin ^{2} \phi+b^{2} \cos ^{2} \phi\right)^{-1 / 4} a \sin \phi \frac{a b^{2} k^{2} q}{\left(k^{2}+v-v k^{2}\right) E(k)-v\left(1-k^{2}\right) K(k)}
$$


where $K(k)$ is the complete elliptic integral of the first kind. Figure 7.3(e) compares the numerical and analytical solutions of two normalized stress intensity factors. $K_{I}$ is normalized by the mean value along the

crack front, $K_{I, \text { mean }}$. $K_{I}$ is normalized by the maximum value along the crack front, $K_{I I, \text { max }}$. The analytical solution is shown for the full elliptical front $\left(-180^{\circ} \leq \phi \leq 180^{\circ}\right)$, while the numerical solution is shown only for the simulated half of the crack front $\left(-90^{\circ} \leq \phi \leq 90^{\circ}\right)$. Overall, the numerical results follow the trends of the analytical solution quite well. The slight fluctuations associated with the numerical results are in part attributable to the tip treatment in the XFEM module. Additionally, it is also noted that $K_{I, \text { mean }}$ and $K_{I I, \max }$ have some errors compared with the analytical results. These errors may be caused by mesh density and interaction integral evaluation in the numerical framework.

The second task is to examine the non-planar crack propagation. Theoretically, when growing, the inclined elliptical crack will continuously make turns and flatten out, eventually creating a partly closeto-horizontal crack for maximized energy release rate under the vertically applied tensile loading. The "flattening-out" behavior has been observed in previous XFEM-based numerical studies [36, 38]. Indeed, with the newly implemented 3D non-planar crack propagation capability, as shown in Figure 7.3(f), the crack evolution predicted by MOOSE agrees with the theoretical expectations and previous numerical studies (Figure 7.3[]g]) quite well. Moreover, numerical results also reveal that, as the crack grows and turns, $K_{I}$ becomes more and more dominant. That is, while $K_{I}$ keeps increasing with the crack growth, changes in $K_{I I}$ are much less significant, making mode-I increasingly important.

\subsection{Summary and Future Work}

The capabilities for modeling crack propagation in the MOOSE XFEM model have been expanded in two important areas: (1) arbitrary non-planar crack growth is now permitted for 3D simulations, and (2) an initial capability for subcritical crack growth has been implemented, specifically for fatigue crack growth using a Paris law. This module now provides the foundational capabilities needed for arbitrary fracture propagation in mechanics problems, which opens up the possibilities for a number of applications of interest for LWR and and advanced reactor structural materials applications. Now that these basic capabilities are available, applications to these problems of interest can begin. Specific applications of interest for LWRs include stresscorrosion cracking in structural components such as boiling water reactor core shrouds. Advanced reactor applications include modeling crack growth in reactor vessels or graphite components. Such applications will require the development of additional models for crack growth in specific materials and under specific conditions that will build on the foundations developed here. 


\section{Acknowledgments}

The high-temperature component modeling work summarized here was the result of collaborations with researchers at Los Alamos National Laboratory (L. Capolungo, A. E. Tallman, M. Arul Kumar) and Argonne National Laboratory (M. C. Messner and A. E. Chakraborty).

The submitted manuscript has been authored by a contractor of the U.S. Government under Contract DE-AC07-05ID14517. Accordingly, the U.S. Government retains a non-exclusive, royalty free license to publish or reproduce the published form of this contribution, or allow others to do so, for U.S. Government purposes.

This research made use of the resources of the High Performance Computing Center at Idaho National Laboratory, which is supported by the Office of Nuclear Energy of the U.S. Department of Energy and the Nuclear Science User Facilities under Contract No. DE-AC07-05ID14517. 


\section{Bibliography}

[1] G.R. Odette and G. E. Lucas. Embrittlement of nuclear reactor pressure vessels. JOM, 53:18-22, 2001.

[2] Huibin Ke, Peter Wells, Philip D. Edmondson, Nathan Almirall, Leland Barnard, G. Robert Odette, and Dane Morgan. Thermodynamic and kinetic modeling of $\mathrm{Mn}-\mathrm{Ni}-\mathrm{Si}$ precipitates in low-Cu reactor pressure vessel steels. Acta Materialia, 138:10 - 26, 2017.

[3] Vitaly V. Slezov. Kinetics of First-order Phase Transitions, chapter 2, pages 7-38. John Wiley \& Sons, Ltd, 2009.

[4] Cluster dynamics code for evolution of Mn-Ni-Si in low-cu RPV steels. https://github.com/ uw-cmg/MNS_CD.

[5] Benjamin W. Spencer, William M. Hoffman, Daniel Schwen, and Sudipta Biswas. Progress on grizzly development for reactor pressure vessels and reinforced concrete structures. 92019.

[6] G.R. Odette, T.Yamamoto, and D. Klingensmith. On the effect of dose rate on irradiation hardening of rpv steels. Philosophical Magazine, 85(4-7):779-797, 2005.

[7] Jia-Hong Ke, Huibin Ke, G. Robert Odette, and Dane Morgan. Cluster dynamics modeling of Mn-Ni-Si precipitates in ferritic-martensitic steel under irradiation. Journal of Nuclear Materials, 498:83 - 88, 2018.

[8] G.R. Odette, T. Yamamoto, T.J. Williams, R.K. Nanstad, and C.A. English. On the history and status of reactor pressure vessel steel ductile to brittle transition temperature shift prediction models. Journal of Nuclear Materials, 526:151863, 2019.

[9] P.T. Williams, T.L. Dickson, B. R. Bass, and H. B. Klasky. Fracture Analysis of Vessels - Oak Ridge, FAVOR, v16.1, computer code: Theory and implementation of algorithms, methods, and correlations. Technical Report ORNL/LTR-2016/309, Oak Ridge National Laboratory, Oak Ridge, TN, September 2016.

[10] B.W. Spencer, W.M. Hoffman, and M.A. Backman. Modular system for probabilistic fracture mechanics analysis of embrittled reactor pressure vessels in the Grizzly code. Nuclear Engineering and Design, 341:25-37, January 2019.

[11] B. W. Spencer, W. M. Hoffman, S. Biswas, and A. Jain. Assessment of Grizzly capabilities for reactor pressure vessels and reinforced concrete structures. Technical Report INL/EXT-20-59941, Idaho National Laboratory, September 2020.

[12] H. F. Bückner. A novel principle for the computation of stress intensity factors. Z. angew Math. Mech., 50:529-546, 1970. 
[13] ASME Boiler and Pressure Vessel Code, Section XI, Rules for Inservice Inspection of Nuclear Power Plant Components. ASME, 2013.

[14] R. C. Cipolla. Computational method to perform the flaw evaluation procedure as specified in the asme code, section xi, appendix a. Technical Report EPRI Report NP-1181, Failure Analysis Associates, September 1979.

[15] Katsumasa Miyazaki, Fuminori Iwamatsu, Shin Nakanishi, and Masaki Shiratori. Stress Intensity Factor Solution for Subsurface Flaw Estimated by Influence Function Method. In ASME Pressure Vessels and Piping Conference, number PVP2006-ICPVT-11-93138, Vancouver, BC, Canada, July 23-27 2006. ASME.

[16] Steven X. Xu, Russell C. Cipolla, Darrell R. Lee, and Douglas A. Scarth. Improvements in Article A-3000 of Appendix A for Calculation of Stress Intensity Factor in Section XI of the 2015 Edition of ASME Boiler and Pressure Vessel Code. Journal of Pressure Vessel Technology, 139(1):010801, August 2016.

[17] ASME Boiler and Pressure Vessel Code, Section XI, Rules for Inservice Inspection of Nuclear Power Plant Components. Number ASME BPVC.XI-2017. ASME, 2017.

[18] Kai Lu, Mano Akihiro, Jinya Katsuyama, Yinsheng Li, and Fuminori Iwamatsu. Development of Stress Intensity Factors for Subsurface Flaws in Plates Subjected to Polynomial Stress Distributions. Journal of Pressure Vessel Technology, 140(3), June 2018.

[19] G. Mehlhorn, J. Kollegger, M. Keuser, and W. Kolmar. Nonlinear contact problems - a finite element approach implemented in adina. Computers and Structures, 21:69-80, 1985.

[20] R. Hameed, A. Turatsinze, F. Duprat, and Sellier A. Bond stress-slip behavior of reinforcing bars embedded in hybrid fiber-reinforced concrete. KSCE:Journal of Civil Engineering, 17:1700-1701, January 2013.

[21] L. B. Munday, S. L. Dhulipala, A. Casagranda, S. A. Pitts, B. W. Spencer, L. Capolungo, A. E. Tallman, M. Arul Kumar, C. Matthews, M. C. Messner, and A. E. Chakraborty. Multiscale-informed modeling of high temperature component response with uncertainty quantification. Technical Report INL/EXT20-59795, Idaho National Laboratory, August 2020.

[22] A. Casagranda, S. A. Pitts, B. W. Spencer, A. Chakraborty, M. C. Messner, and L. Capolungo. Evaluation of high temperature material models for high temperature advanced reactor component analysis. In Proceedings of ASME 2020 Pressure Vessels and Piping Conference, Paper No. 21614, Minneapolis, Minnesota, July 2020.

[23] Hervé Moulinec and Pierre Suquet. A numerical method for computing the overall response of nonlinear composites with complex microstructure. Computer methods in applied mechanics and engineering, 157(1-2):69-94, 1998.

[24] Dana A Knoll and David E Keyes. Jacobian-free newton-krylov methods: a survey of approaches and applications. Journal of Computational Physics, 193(2):357-397, 2004.

[25] Ricardo A Lebensohn. N-site modeling of a 3d viscoplastic polycrystal using fast fourier transform. Acta materialia, 49(14):2723-2737, 2001.

[26] TWJ De Geus, J Vondřejc, J Zeman, RHJ Peerlings, and MGD Geers. Finite strain fft-based non-linear solvers made simple. Computer Methods in Applied Mechanics and Engineering, 318:412-430, 2017. 
[27] Jan Zeman, Tom WJ de Geus, Jaroslav Vondřejc, Ron HJ Peerlings, and Marc GD Geers. A finite element perspective on nonlinear fft-based micromechanical simulations. International Journal for Numerical Methods in Engineering, 111(10):903-926, 2017.

[28] Sergio Lucarini and Javier Segurado. On the accuracy of spectral solvers for micromechanics based fatigue modeling. Computational Mechanics, 63(2):365-382, 2019.

[29] Cody J Permann, Derek R Gaston, David Andrš, Robert W Carlsen, Fande Kong, Alexander D Lindsay, Jason M Miller, John W Peterson, Andrew E Slaughter, Roy H Stogner, et al. Moose: Enabling massively parallel multiphysics simulation. SoftwareX, 11:100430, 2020.

[30] Wen Jiang, Benjamin W. Spencer, and John E. Dolbow. Ceramic nuclear fuel fracture modeling with the extended finite element method. Engineering Fracture Mechanics, 223:106713, January 2020.

[31] B. W. Spencer, S. A. Pitts, L. Liu, M. Vyas, W. Jiang, A. Casagranda, and D. J. McDowell. Summary of Grizzly development for advanced reactor structural materials. Technical Report INL/EXT-19-55958, Idaho National Laboratory, September 2019.

[32] Yahya Ali Fageehi and Abdulnaser M. Alshoaibi. Numerical simulation of mixed-mode fatigue crack growth for compact tension shear specimen. Advances in Materials Science and Engineering, 2020:114, April 2020.

[33] Keisuke Tanaka. Fatigue crack propagation from a crack inclined to the cyclic tensile axis. Engineering Fracture Mechanics, 6(3):493-507, October 1974.

[34] N. Moës, A. Gravouil, and T. Belytschko. Non-planar 3D crack growth by the extended finite element and level sets-Part I: Mechanical model. International Journal for Numerical Methods in Engineering, 53(11):2549-2568, April 2002.

[35] R. Singh, B.J. Carter, P.A. Wawrzynek, and A.R. Ingraffea. Universal crack closure integral for SIF estimation. Engineering Fracture Mechanics, 60(2):133-146, May 1998.

[36] N. Sukumar, D. L. Chopp, E. Béchet, and N. Moës. Three-dimensional non-planar crack growth by a coupled extended finite element and fast marching method. International Journal for Numerical Methods in Engineering, 76(5):727-748, October 2008.

[37] M. K. Kassir and G. C. Sih. Three-dimensional stress distribution around an elliptical crack under arbitrary loadings. Journal of Applied Mechanics, 33(3):601-611, September 1966.

[38] Himanshu Pathak, Akhilendra Singh, and Indra Vir Singh. Fatigue crack growth simulations of 3-D problems using XFEM. International Journal of Mechanical Sciences, 76:112-131, November 2013. 